\title{
Fibrosis Rescue Improves Cardiac Function in Dystrophin-Deficient Mice and Duchenne Patient-Specific Cardiomyocytes by Immunoproteasome Modulation
}

Andrea Farini, ${ }^{*}$ Aoife Gowran, ${ }^{\dagger}$ Pamela Bella, ${ }^{*}$ Clementina Sitzia, ${ }^{\dagger}$ Alessandro Scopece, ${ }^{\dagger}$ Elisa Castiglioni, ${ }^{\dagger}$ Davide Rovina, ${ }^{\dagger}$ Patrizia Nigro, ${ }^{\dagger}$ Chiara Villa, ${ }^{*}$ Francesco Fortunato, ${ }^{\S}$ Giacomo Pietro Comi, ${ }^{\S}$ Giuseppina Milano, ${ }^{\dagger \uparrow}$ Giulio Pompilio, $\|_{* * \dagger \dagger}$ and Yvan Torrente*

\begin{abstract}
From the Stem Cell Laboratory, * Department of Pathophysiology and Transplantation, Universitá degli Studi di Milano, Unit of Neurology, Fondazione IRCCS Ca' Granda Ospedale Maggiore Policlinico, Centro Dino Ferrari, Milan, Italy; the Unit of Vascular Biology and Regenerative Medicine, ${ }^{\dagger}$ Centro Cardiologico Monzino-IRCCS, Milan, Italy; the UOC SMEL-1, ${ }^{\ddagger}$ Scuola di Specializzazione di Patologia Clinica e Biochimica Clinica, Università degli Studi di Milano, Milan, Italy; the Neurology Unit, ${ }^{\S}$ Neuroscience Section, Department of Pathophysiology and Transplantation, Dino Ferrari Centre, IRCCS Foundation Ca' Granda Ospedale Maggiore Policlinico, University of Milan, Milan, Italy; the Laboratory of Cardiovascular Research, Department of Surgery and Anesthesiology, University Hospital of Lausanne, Lausanne, Switzerland; the Unit of Vascular Biology and Regenerative Medicine," the Department of Cardiac Surgery, ${ }^{* *}$ Centro Cardiologico Monzino-IRCCS, Milan, Italy; and the Department of Clinical Sciences and Community Health, ${ }^{\dagger \dagger}$ University of Milan, Milan, Italy
\end{abstract}

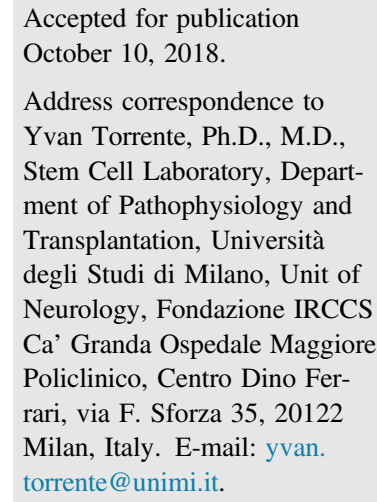

\begin{abstract}
Patients affected by Duchenne muscular dystrophy (DMD) develop a progressive dilated cardiomyopathy characterized by inflammatory cell infiltration, necrosis, and cardiac fibrosis. Standard treatments consider the use of $\beta$-blockers and angiotensin-converting enzyme inhibitors that are symptomatic and unspecific toward DMD disease. Medications that target DMD cardiac fibrosis are in the early stages of development. We found immunoproteasome dysregulation in affected hearts of $m d x$ mice (murine animal model of DMD) and cardiomyocytes derived from induced pluripotent stem cells of patients with DMD. Interestingly, immunoproteasome inhibition ameliorated cardiomyopathy in $m d x$ mice and reduced the development of cardiac fibrosis. Establishing the immunoproteasome inhibition-dependent cardioprotective role suggests the possibility of modulating the immunoproteasome as new and clinically relevant treatment to rescue dilated cardiomyopathy in patients with DMD. (Am J Pathol 2019, 189: 339-353; https://doi.org/10.1016/ j.ajpath.2018.10.010)
\end{abstract}

Skeletal myopathy and muscular dystrophy progression are commonly associated with cardiac dysfunctions and a consequent high mortality attributable to heart failure. ${ }^{1-3}$ In particular, patients with Duchenne muscular dystrophy (DMD) present with early diastolic dysfunction and myocardial fibrosis that turn into a dilated cardiomyopathy, complicated by heart failure and arrhythmia. ${ }^{4}$ Even though recent improvements in the management of respiratory insufficiency have improved the lifespan and overall prognosis of patients with DMD, sudden deaths attributable to heart failure negatively affect their quality of life. Prompt treatment and early detection of cardiomyopathy represent the requirements for successful cardioprotective therapies that block or at least slow the processes of cardiac remodeling and heart failure. ${ }^{3}$ Unfortunately, the current treatments for dilated cardiomyopathy are still inadequate because a deep understanding of the specific mechanisms underlying DMD-attributable heart failure is

\footnotetext{
A.F. and A.G. contributed equally to this work.

Supported by Italian Ministry of Health Conte Capitale 2015 (G.P.), Fondazione Umberto Veronesi (A.G.), and Fondazione IEO CCM (D.R.), Disclosures: None declared.
} 
lacking. Common approaches are standard and rely on the use of angiotensin-converting enzyme inhibitors and $\beta$-adrenoceptor antagonists. ${ }^{3}$ Most patients with DMD develop cardiomyopathic features between 10 and 15 years of age. Because of this tight timeline during which heart dysfunction appears, DMD offers a unique opportunity to assess strategies to limit cardiomyopathy progression. In the heart of the DMD murine animal model (the $m d x$ mouse), from 8 weeks of age, the loss of dystrophin and membrane integrity affects $\mathrm{Ca}^{2+}$ handling and nitric oxide signaling ${ }^{5}$ so that $m d x$ cardiomyocytes are susceptible to mechanical stress-induced contractile failure and necrosis. ${ }^{6}$ Similarly, in skeletal muscle, the lack of dystrophin determines the pathological infiltration of immune cells, such as $\mathrm{T}$ lymphocytes and macrophages, and the release of inflammatory cytokines, activating NF- $\kappa \mathrm{B}-$ dependent pathways. ${ }^{7}$ Dilated cardiomyopathy and contractile deviances are evident in 36- to 40-week-old $m d x$ mice. $^{8}$

Cardiac stress has been recognized for its main role in the up-regulation of inflammatory cytokines and growth factors and the generation of reactive oxygen species, which modulate different signaling cascades, whose dysfunctions cause altered cytokine secretion and fibrosis that commonly affects dystrophic hearts. In particular, the Janus kinase (JAK) STAT can transduce the signal of IL-6, IL-10, and interferon- $\gamma$ by means of the selective phosphorylation of STAT1/3. ${ }^{9}$ Similarly, STAT phosphorylation can be driven by different serine kinases, such as p38 mitogen-activated protein kinase and extracellular-regulated kinase (ERK). ${ }^{9}$ In addition, STATs together with $\mathrm{NF}-\kappa \mathrm{B}$ synergistically up-regulate inducible nitric oxide synthase in DMD muscles, causing the inhibition of the soluble guanylate cyclase $1 \alpha,{ }^{10}$ whereas STAT3 interacts with osteopontin in extracellular matrix development. ${ }^{11}$ A murine model of hyperglycemia found that the proliferative capacity of cardiac fibroblasts and their ability to express collagen was regulated by STAT1/3 possibly through phosphorylated ERK1/2 blockade of collagen expression. ${ }^{12}$ Intriguingly, most of these proteins and cytokines are deregulated in patients with DMD and their functions are commonly influenced by the immunoproteasome.

The immunoproteasome is formed by the replacement of the catalytic subunits of the constitutive proteasome with other subunits, termed PSMB8, PSMB9, and PSMB10, that are induced by inflammatory stimuli, such as tumor necrosis factor $\alpha(\mathrm{TNF}-\alpha)$ and interferon- $\gamma$. Moreover, it is a critical regulator of $\mathrm{NF}-\kappa \mathrm{B}$ signaling driven by selective phosphorylation of STAT1/3. ${ }^{9}$ In the cells of the immune system, the highly expressed immunoproteasome plays a role in generating peptide ligands for major histocompatibility complex class I antigen presentation. ${ }^{13}$ The immunoproteasome is also present in the heart and is overexpressed in dystrophic muscles. ${ }^{14}$ Immunoproteasome up-regulation was observed with concomitant loss of cardiac muscle mass, ${ }^{15}$ inflammation, ${ }^{16}$ and myocyte atrophy ${ }^{17}$ and, in contrast, with overexpression of oxygen species and development of atrial fibrosis. ${ }^{18}$
So far, there are several promising immunoproteasome inhibitors that have been developed and already assessed in phase $1 / 2$ of clinical trials for selectively treating patients with inflammatory and autoimmune diseases. ${ }^{19,20}$ Of note, the potent inhibitor ONX-0914, originally named PR957 and specifically targeted toward the highly active subunit LMP7 ( $\beta 5 \mathrm{i})$ of the immunoproteasome, ${ }^{21}$ was successfully used to treat a viral myocarditis by diminishing the expression of proinflammatory cytokines and chemokines, reducing infiltration of inflammatory cells, and leading to a general improvement of the cardiac output. ${ }^{22}$ In line with this result, our previous work found that ONX-0914 treatment modulates dystrophic features in $m d x$ mice by reducing the amount of infiltrating activated $\mathrm{T}$ cells, myofiber necrosis, and collagen deposition in skeletal muscle tissues. $^{23}$

The present study confirmed the possibility of using ONX-0914 for inhibiting the immunoproteasome function, therefore countervailing inflammatory cells and fibrosis in dilated cardiomyopathy of $m d x$ mice and improving their hemodynamic performance. ONX-0914 treatment dampened the release of proinflammatory cytokines, decreased major histocompatibility complex I expression, and increased anti-inflammatory $\mathrm{FoxP}^{+}$regulatory $\mathrm{T}$ lymphocytes. Considering that early dilated cardiomyopathy is characterized by mild left ventricular dysfunction and differs from advanced dilated cardiomyopathy for left ventricular systolic and diastolic dysfunction, the effect of ONX-0914 treatment was tested in $m d x$ mice with early (6-week-old) and advanced (9-month-old) dilated cardiomyopathy. Interestingly, ONX-0914 could both counteract the raising of the symptoms of cardiomyopathy in younger $m d x$ mice and alleviate the pathological findings in older $m d x$ mice.

Our study underlines the substantial contribution of the immunoproteasome in infiltrating myocardial immune cells, which actively participate in cardiomyocyte death and successive fibrosis of the DMD heart. Furthermore, the data were translated from $m d x$ mice to human cardiomyocytes derived from the induced pluripotent stem cells (iPSCs) of patients with DMD, which also displayed aberrant involvement of the immunoproteasome pathway. On the basis of the present and the previous findings, ${ }^{23}$ we suggest that the immunoproteasome possesses a key role directly involved in the poor clinical outcomes observed in patients with DMD, therefore representing a promising candidate target for rescuing dystrophic dilated cardiomyopathy.

\section{Materials and Methods}

\section{Animal Ethics Statement}

All the procedures performed on living animals comply with Italian law (D.L.vo 116/92 and subsequent additions) and are approved by local ethics committees. This work was authorized by the Ministry of Health and Local University of Milan Committee (authorization number 859/2017-PR, 
Table 1 List of Primers for Quantitative RT-PCR

\begin{tabular}{ll}
\hline Primer name & Primer sequence \\
\hline m-TNF- $\alpha$ & \\
Forward & $5^{\prime}$-CTACCTTGTTGCCTCCTCTTT-3' \\
Reverse & $5^{\prime}$-GAGCAGAGGTTCAGTGATGTAG-3' \\
m-IL-1 $\beta$ & \\
Forward & $5^{\prime}$-TCTGATGGGCAACCACTTAC-3' \\
Reverse & $5^{\prime}$-GTTGACAGCTAGGTTCTGTTCT-3' \\
m-CCL2 & \\
Forward & $5^{\prime}$-TTTCTTAAATGCAAGGTGTGGA-3' \\
Reverse & $5^{\prime}$-CCTTGGAATCTCAAACACAAAGT-3' \\
m-ICAM-1 & \\
Forward & $5^{\prime}$-AGTAGATCAGTGAGGAGGTGAA-3' \\
Reverse & $5^{\prime}$-GCATCCTGACCAGTAGAGAAAC-3' \\
m-R0R- $\gamma$ t & \\
Forward & $5^{\prime}$-GACTGACAATCAGCAGGGATAA-3' \\
Reverse & $5^{\prime}$-GGGAAATACAATGAGGTATTGAAAGG-3' \\
COL3A & \\
Forward & $5^{\prime}$-GCCTTCTACACCTGCTCCTG-3' \\
Reverse & $5^{\prime}$-GATCCAGGATGTCCAGAGG-3' \\
\hline
\end{tabular}

CCL2, chemokine ( $C-C$ motif) ligand 2; COL3A, collagen 3A; ICAM-1, intercellular adhesion molecule $1 ; \mathrm{m}-$, mouse-specific; ROR- $\gamma \mathrm{t}$, retinoic acid receptor-related orphan receptor $\gamma$; TNF- $\alpha$, tumor necrosis factor $\alpha$.

5247B.35, 10/07/2017). Six-week-old, male, wild-type (C57Bl) mice, 6-week-old $m d x$ mice, and 9-month-old $m d x$ mice were provided by Charles River Laboratories (Wilmington, MA). All animals were housed in a controlled ambient environment (12-hour light/dark cycle) at a temperature between $21^{\circ} \mathrm{C}$ and $23^{\circ} \mathrm{C}$. The mice had free access to clean water and food. Intraperitoneal injection of the immunoproteasome inhibitor ONX-0914 (6 mg/kg; CliniSciences, Nanterre, France) was performed on 6-week-old and 9-month-old $m d x$ mice for 5 weeks (two injections per week, $n=10$ ). Untreated, aged-matched $m d x$ mice were used as controls.

\section{iPSC Generation and Characterization}

All investigations were conducted after informed consent was provided under regulation of local ethics committee approval (Centro Cardiologico Monzino, Milan, Italy). Human fibroblasts were isolated from the skin biopsy specimens of patients with DMD. Healthy adult dermal fibroblasts were obtained from tebu-bio (Le-Perray-en-Yvelines, France) and served as controls. Fibroblasts were culture expanded in Dulbecco's modified Eagle's medium supplemented with 10\% HyClone fetal bovine serum (GE Healthcare Life Sciences, Buckinghamshire, UK), $1 \times$ MEM Non-Essential Amino Acid Solution, $2 \mathrm{mmol} / \mathrm{L}$ L-glutamine (both from Stemcell Technologies, Vancouver, Canada), and basic fibroblast growth factor. To generate iPSCs, fibroblasts were transfected with four episomal vectors (pCXLE-hUL, pCXLE-hSK, pCXLE-hOCT3/4-shp53-F, and a positive control pCXLE-EGFP) by electroporation $(1650 \mathrm{~V}, 10 \mathrm{~ms}, 3$ pulses) with the Neon transfection system (Invitrogen,
Carlsbad, CA). Transfected fibroblasts were grown on human recombinant vitronectin-coated multiwell plates and maintained in solution for 48 hours at which point the media was changed to TeSR reprogramming media (Stemcell Technologies) with daily media changes. Emergent iPSC colonies were manually isolated between posttransfection days 21 to 30 with a 25-G syringe and seeded onto vitronectin-coated multiwell plates in mTeSR1 media (Stemcell Technologies). Fresh medium was replaced daily. From postoperative day 4 onward iPSCs were passaged without the use of enzymes every 3 to 4 days with ReLeSR (Stemcell Technologies) and plated as cell aggregates onto vitronectin-coated multiwell plates. Alkaline phosphatase activity was detected in iPSCs after incubation with Alkaline Phosphatase Live Stain (Invitrogen) for 30 minutes at $37^{\circ} \mathrm{C}$. After washing, fluorescent-labeled colonies were visualized with a fluorescein isothiocyanate (FITC) filter and $20 \times$ objective (ApoTome, Zeiss, Oberkochen, Germany). For the analysis of pluripotency protein expression, stage-specific embryonic antigen-4 (SSEA4) was detected using a commercially available antibody (mouse anti-SSEA4, 1:100 in 5\% normal goat serum overnight at $4{ }^{\circ} \mathrm{C}$; Abcam, Cambridge, UK) and revealed by Alexa Fluor 488 anti-mouse secondary antibody (1:400). Nuclei were counterstained with Hoechst 33,342 (Invitrogen). iPSCs were analyzed with FITC filter and 20x objective (LSM710; Zeiss). For FACS analyses, iPSCs were gently dissociated using cell dissociation reagent (Stemcell Technologies) and stained with Tra-1-60 (1:100, 1 hour; Abcam). Five percent bovine serum albumin (BSA; SigmaAldrich, St. Louis, MO) in phosphate-buffered saline was used as blocking solution for excluding nonspecific staining (Lonza, Caravaggio, Italy). Goat anti-mouse IgM FITC (1:200, 1 hour; Life Technologies, Carlsbad, CA) was exploited as secondary antibody. Cells were analyzed using a FACSCalibur (BD Biosciences, Franklin Lakes, NJ) or Gallios (Beckman Coulter Life Sciences, Indianapolis, IN) flow cytometers.

\section{iPSC Cardiomyocyte Differentiation and Characterization}

Cardiomyocyte differentiation of iPSCs was performed following the Lian et $\mathrm{al}^{24}$ monolayer-directed cardiomyocyte differentiation protocol. Briefly, on day 0 of differentiation, iPSCs were treated with a GSK3 inhibitor [12 $\mu \mathrm{mol} / \mathrm{L}$ CHIR99021 in RPMI 1640 medium supplemented with insulin-free B27 (Selleck Chemicals LLC, Houston, TX, and Invitrogen, respectively)]. The media was replaced with RPMI supplemented with insulin-free B27 after 24 hours. On day 3, a combined medium was prepared that contained $1 \mathrm{~mL}$ of conditioned media and $1 \mathrm{~mL}$ of $10 \mu \mathrm{mol} / \mathrm{L}$ IWP2 (a Wnt signaling inhibitor, final concentration of 5 $\mu \mathrm{mol} / \mathrm{L}$ ) in RPMI supplemented with insulin-free B27. On day 5, the combined medium was replaced with RPMI supplemented with insulin-free B27. On day 7, the medium was changed to RPMI supplemented with B27 containing insulin. 

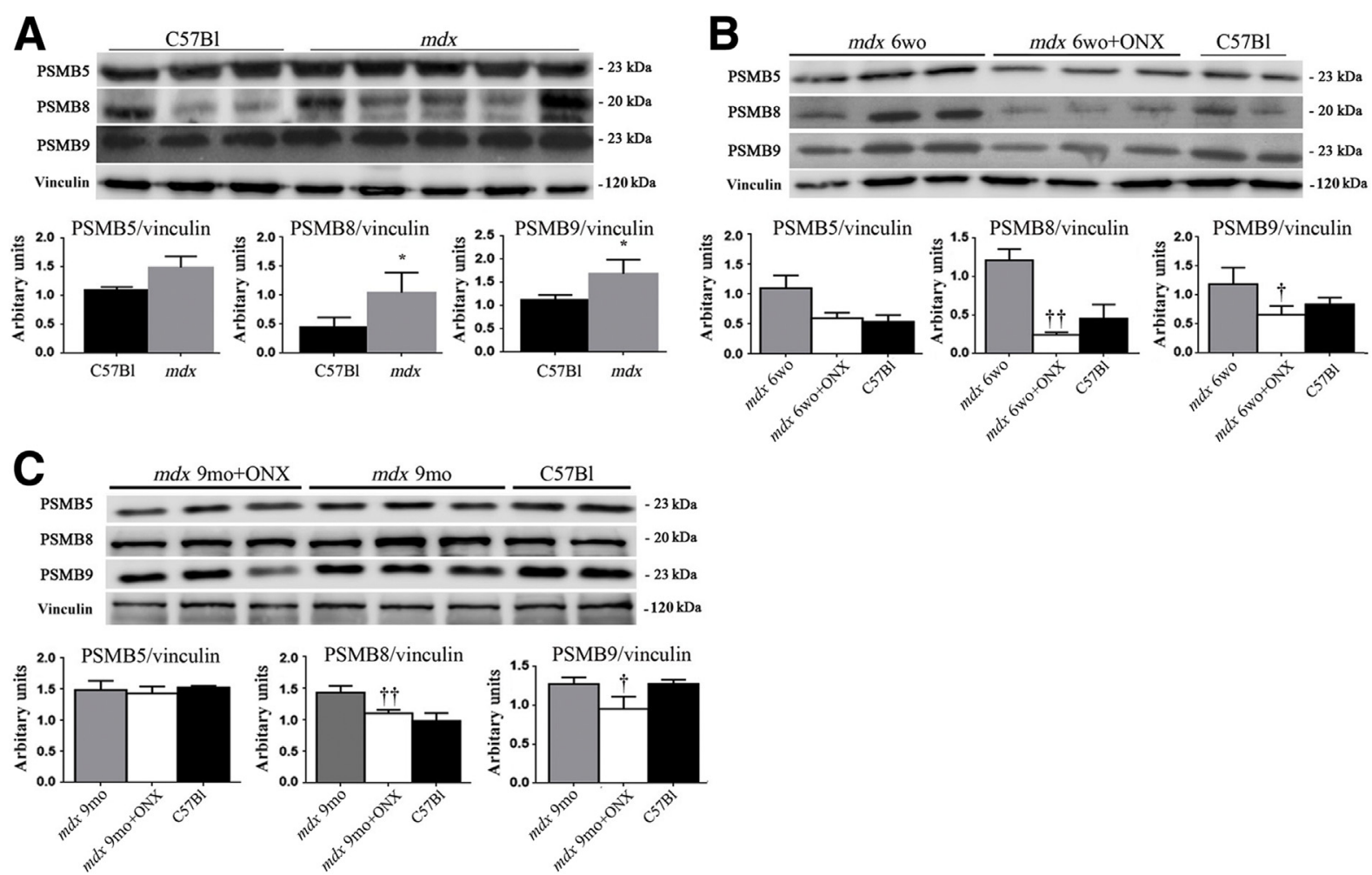

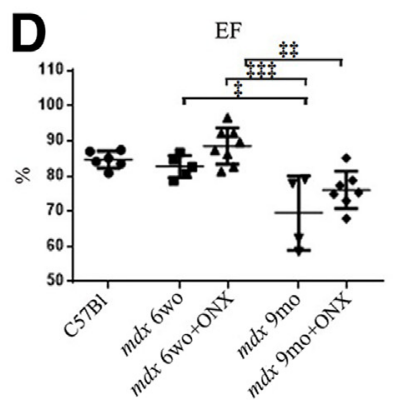

LVAWd

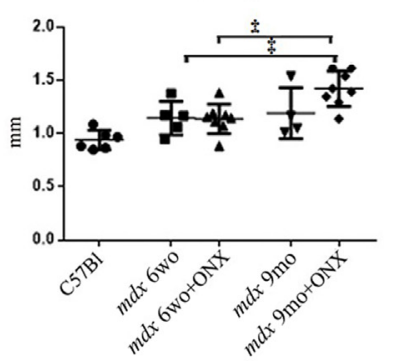

E
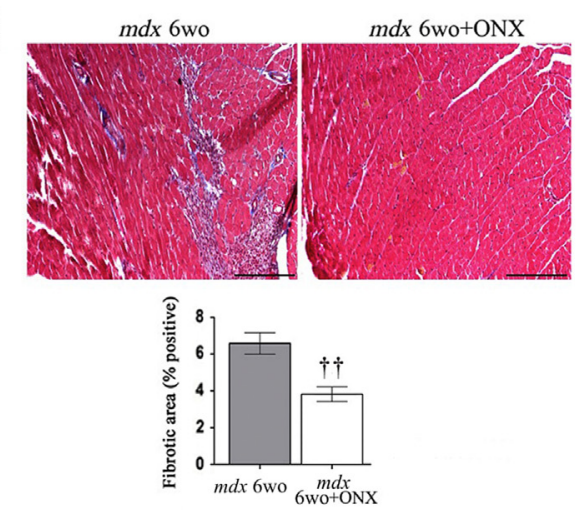

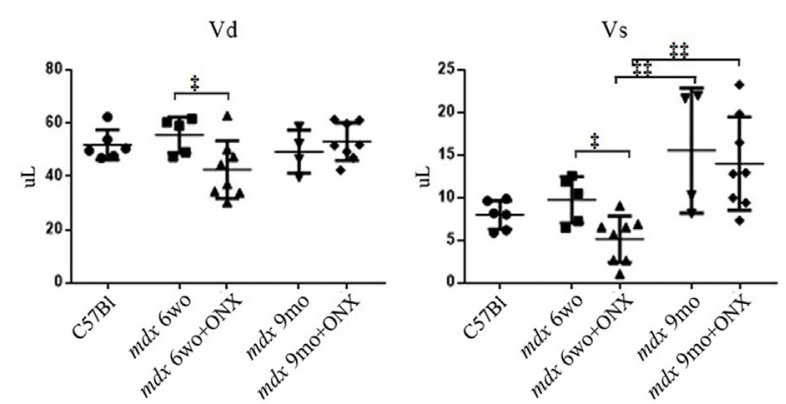

LVIDd

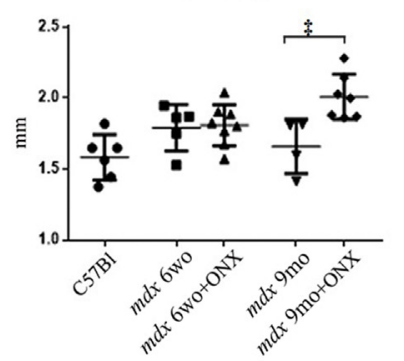

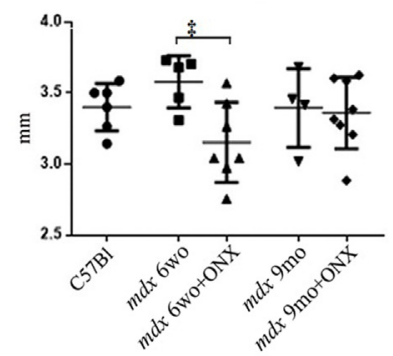

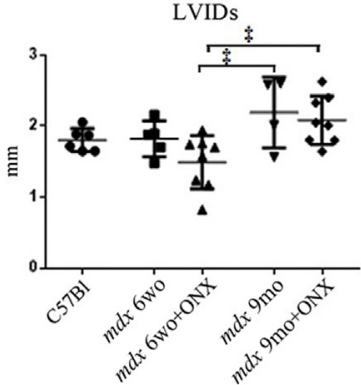

F
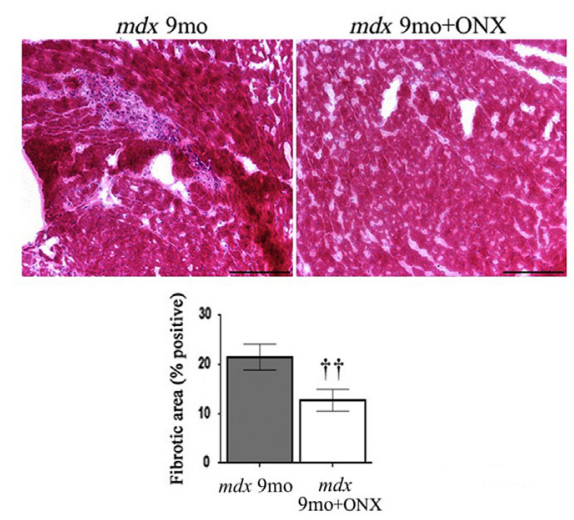
From this point, the medium was changed every 3 days. After 16 days in culture, cardiomyocytes derived from human iPSCs (CMs-d-iPSCs) were processed for further analysis. For immunofluorescence analyses of cardiac troponin $\mathrm{T}$ type 2 (cTnT2), a commercial cardiomyocyte characterization kit (Life Technologies) was used following the manufacturer's instructions, with the exception of a secondary antibody that was conjugated to Alexa Fluor 633 (Life Technologies). Cell dissociation reagent (Stemcell Technologies) was used for gently dissociating CM-d-iPSCs for flow cytometry analysis. Afterward, cells underwent fixation, permeabilization (BD Biosciences), and blocking using 5\% BSA (Sigma-Aldrich) in phosphate-buffered saline (Lonza). Cells were stained with an anti-cTnT2 antibody (1:100, 1 hour; Life Technologies) followed by goat anti-mouse IgG1 FITC (1:200, 1 hour; Life Technologies). Cells were analyzed using a FACS Calibur flow cytometer (BD Biosciences). After 14 days of differentiation, CMs-d-iPSCs were harvested and plated onto fibronectin-coated multielectrode array plates. Cardiac depolarization and repolarization, $\mathrm{T}$ waves, and field potential durations were detected with the Maestro multielectrode array system's cardiac beat detector data processor (Axion BioSystems, Atlanta, GA). Mean field potential durations were obtained with the Comprehensive in Vitro Proarrhythmia Assay analysis tool version 9.2 (Axion BioSystems) and plotted using the AxIS Metric plotting Tool (Axion BioSystems).

\section{Modeling DMD Cardiomyopathy with Patient-Specific CMs-d-iPSCs}

The determination of dystrophin protein expression in CMs-diPSCs by Western blotting was performed by standard techniques using an anti-dystrophin antibody that recognizes human dystrophin (1:500; Abcam) and an anti-glyceraldehyde-3phosphate dehydrogenase antibody as a housekeeping protein (1:2000; Abcam). Intracellular $\mathrm{Ca}^{2+}$ was determined with a Fluo-4 Calcium Imaging Kit immunofluorescence-based assay kit (Life Technologies) and random images were captured with an ApoTome (Zeiss). Pixel intensity or percent area were calculated using ImageJ software version 1.51i (NIH, Bethesda, MD; http://imagej.nih.gov/ij). cTnI or TNF- $\alpha$ in CMs-diPSC-conditioned media was measured with commercial enzyme-linked immunosorbent assay kits (Life Technologies) and normalized to total protein.

\section{Histologic Analysis}

Animals were sacrificed by cervical dislocation, and cardiac muscles of ONX-0914 treated and untreated $m d x$ mice were collected for both histologic and biochemical analyses. Muscles destined to collagen staining were frozen in liquidnitrogen-cooled isopentane and cut on a cryostat (Leica CM1850) into $8 \mu \mathrm{m}$. Staining of sections was performed with rabbit anti-collagen 1 antibody (COL1A; Cell Signaling Technology, Danvers, MA). Briefly, heart tissues were blocked for 60 minutes with 3\% BSA in phosphate-buffered saline at room temperature and then incubated overnight at $4^{\circ} \mathrm{C}$ with primary antibody diluted 1:300 in blocking solution. Monoclonal anti-rabbit 594 (Molecular Probe, Invitrogen) was added at a dilution of 1:200 in blocking solution for 1 hour. Nuclei were stained with DAPI (Sigma-Aldrich), and images were captured with a Leica TCS SP2 confocal microscope. Before sacrificed, other animals were subjected to cardiac perfusion with saline followed by a $10 \%$ formalin flush. To determine the amount of fibrosis, cardiac tissues were stained by Azan Mallory: the percentage of collagen area per section (stained in blue) was measured by ImageJ.

\section{Western Blot Analysis}

Hearts were isolated from ONX-0914-treated and untreated $m d x$ mice and total protein concentration obtained as previously described. ${ }^{25}$ Samples were resolved on polyacrylamide gels (ranging from 6\% to 12\%) and transferred to nitrocellulose membranes (Bio-Rad Laboratories, Hercules, CA). The membranes were incubated overnight with the primary antibodies against: PSMB5 (1:500; Abcam); PSMB8 (1:500; Abcam); PSMB9 (1:500; Abcam); $\beta$ tubulin III (1:400; Sigma-Aldrich); vinculin (1:1000; Santa Cruz Biotechnology, Santa Cruz, TX); osteopontin (1:500; R\&D Systems, Minneapolis, MN); FoxP3 (1:500; eBioscience, San Diego, CA); TNF- $\alpha$ (1:500; eBioscience); transient receptor potential channel 1 (TRPC1; 1:500; Santa Cruz Biotechnology); STAT1 (1:400; Cell Signaling Technology); phosphorylated STAT1 (1:400; Cell Signaling Technology); STAT-3 (1:400; Cell Signaling Technology); and phosphorylated STAT3 (1:400; Cell Signaling Technology). After incubation, the membranes were detected with peroxidase-conjugated secondary antibodies (Agilent Technologies, Santa Clara, CA) and developed by ECL (Amersham Biosciences, Little Chalfont, UK).

Figure 1 Expression of immunoproteasome (IP) in C57BL, $m d x$, and 0NX-0914-treated $m d x$ mice and evaluation of IP inhibition in $m d x$ mice. A and B: Representative measurement of the expression of IP subunits. Western blotting analysis reveals overexpression of IP subunits PSMB8 and PSMB9 in the hearts of 6-week-old (6wo) $m d x$ mice related to C57Bl (A) and demonstrates that treatment with 0NX-0914 down-regulates IP expression in 6wo mdx mice (B). C: Similar down-regulation of IP subunits amount is evidenced in 9-month-old (9mo) mdx mice treated with 0NX-0914. D: Transthoracic echocardiography reveals that 0NX-0914-treated 6wo $m d x$ mice have a significant decrease of left ventricular end-systolic volume (Vs) and end-diastolic volume (Vd) with significant improvement of LV ejection fraction (EF), whereas 0NX-0914-treated 9mo mdx mice up-regulate stroke volume (SV) and left ventricular anterior wall thickness in systole (LVAWs). E and F: Representative Azan Mallory images of the left ventricle of 0NX-0914-treated and untreated 6wo (E) and 9mo (F) $m d x$ mice. Histogram represents the percentage of cardiofibrotic area per section of treated and untreated $m d x$ mice. Data are expressed as means \pm SEM. $n=3$ independent experiments (A); $n=3$ to 5 independent experiments (B and $\mathbf{C}) ; n=5$ to 8 independent experiments (D). * $P<0.05$ versus $C 57 \mathrm{Bl}(t-$ test); ${ }^{\dagger} P<0.05,{ }^{\dagger \dagger} P<0.01$ versus $m d x$ (t-test); ${ }^{\ddagger} P<0.05,{ }^{\ddagger \ddagger} P<0.01$, and ${ }^{\ddagger \ddagger \ddagger} P<0.001$ (two-way analysis of variance). Scale bars $=200 \mu \mathrm{m}$. LVAWd, left ventricular anterior wall thickness in diastole. 


\section{Mitochondrial Enzyme Analysis}

Cardiac biopsy specimens were collected from ONX 0914-treated and untreated $m d x$ mice, and the samples were prepared for analysis as previously described. ${ }^{26}$ Mitochondrial respiratory chain enzymes and the citrate synthase activities were measured by means of a spectrophotometer as previously described. ${ }^{27}$ The value of citrate synthase was used to normalize the values of the other complexes.

\section{Quantitative RT-PCR Experiments}

Total RNA was extracted from cardiac biopsies obtained from ONX-0914-treated and untreated $m d x$ mice. cDNA was generated using the Reverse Transcriptase Kit (ThermoFisher Scientific) followed by the SYBR-Green method to quantify the expression of the genes listed in Table 1. All the cDNA samples were tested in duplicate, and the threshold cycle $\left(\mathrm{C}_{\mathrm{T}}\right)$ of each target gene was normalized against $G A P D H$, which was considered a housekeeping gene. Relative transcript levels were calculated from the $\mathrm{C}_{\mathrm{T}}$ values as $\mathrm{X}=2^{-\Delta \mathrm{ct}}$, where $\mathrm{X}$ is the fold difference in amount of target gene versus GAPDH and $\Delta \mathrm{C}_{\mathrm{T}}=\mathrm{C}_{\mathrm{Ttarget}}-\mathrm{C}_{\mathrm{TGAPDH}}$.

\section{Image Quantification}

Histochemical staining was imaged with an Axioskop II microscope using a digital camera (AxioCamColor) and Axiovision software version 4.7 (all by Zeiss), a Leica TCS SP2 confocal system, or a Zeiss 710 multiphoton confocal mircroscope with ZEN software version 2010D. Densitometric analyses and manual or automatic counting (Threshold Color Plug-in) were performed using ImageJ software version 2.0.0-rc-2 in 20 sections per muscle.

\section{Statistical Analysis and Power Calculations}

Data were analyzed by GraphPad Prism software version 6 (GraphPad Software, San Diego, CA) and expressed as means \pm SD or means \pm SEM. To compare multiple group means, one-way and two-way analysis of variance was used, and multiplicity adjusted $P$ values for each comparison were reported for each analysis. For the comparison of two groups, a $t$-test was applied assuming equal variances. The difference among groups was considered significant at $P<0.05$.

The number of animals per experiment was calculated with the help of the dedicated G POWER online software version $3.1,{ }^{28}$ considering a power of $80 \%$ and a significance level of $P<0.05$. Based on literature data, the minimum number of animals per experimental group required to observe statistically significant differences is 6 mice per group imaging (ultrasonographic and hemodynamic) and molecular techniques, whereas 4 mice per group are required for immunohistochemical analyses for each timepoint.

\section{Results}

\section{NX-0914 Treatment of $m d x$ Mice Affects Dilated Cardiomyopathy}

Following up on previous work, ${ }^{23}$ the expression of constitutive proteasome and immunoproteasome subunits was analyzed by Western blotting in the hearts of 6-week-old $m d x$ mice and $\mathrm{C} 57 \mathrm{Bl}$ mice. The results revealed that 6-week-old $m d x$ mice overexpressed IP subunits (PSMB8, $P=0.040$; PSMB9, $P=0.0491$ ), whereas the expression of the constitutive proteasome subunit (PSMB5) was not altered (Figure 1A). To test the efficacy of both early- and later-stage treatment on DMD cardiac pathological findings, the effect of ONX-0914 injection was investigated in 6-week-old and 9-month-old $m d x$ mice: ONX-0914 was administered intraperitoneally two times per week in 6-week-old and 9month-old $m d x$ mice for 5 weeks followed by transthoracic echocardiography and morphologic analysis. In the hearts of $m d x$ mice treated with ONX-0914, PSMB8 and PSMB9 expression was down-regulated (PSMB8, $P=0.0184$; PSMB9, $P=0.0437$ ), whereas the amount of PSMB5 was unchanged (Figure 1B). Similarly, in treated 9-month-old $m d x$ mice, PSMB8 and PSMB9 were down-regulated (PSMB8, $P=0.0376$; PSMB9, $P=0.0093$ ), whereas the expression of PSMB5 was unaltered (Figure 1C).

Regarding the transthoracic echocardiography, the aged $m d x$ mice had a significant decrease of the ejection fraction (two-way analysis of variance; $P=0.0189$ ) and in the scale stroke volume (two-way analysis of variance; $P=0.0034$ ). However, ONX-0914 treatment induced a significant agedependent amelioration of ejection fraction (two-way analysis of variance; $P=0.0037$ ) and stability of stroke volume (Figure 1D). The ONX-0914 treatment resulted in a remarkable reduction in left ventricular (LV) end-systolic (two-way analysis of variance; $P=0.0481$ ) and LV enddiastolic (two-way analysis of variance; $P=0.0453$ ) volumes in ONX-0914-treated 6-week-old $m d x$ mice compared with untreated age-matched mice (Figure 1D), Moreover, the ONX-0914 treatment did not affect the LV alterations of 9-month-old $m d x$ mice. In addition, after ONX-0914 treatment, the 6-week-old and 9-month-old $m d x$ mice had significant differences in the length of LV anterior wall thickness in diastole and systole) and in the length of LV internal diameter in diastole and systole (Figure 1D). Other echocardiographic measurements revealed significant impairment of cardiac output $(P=0.0216)$ in aging $m d x$ mice but not in ONX0914-treated age-matched $m d x$ mice. Length of diameter in diastole was stable over time. ONX-0914-treated $m d x$ mice had significant differences at 9 months compared with 6 weeks for length of diameter in systole $(P=0.0022), \mathrm{LV}$ mass $(P=0.0256)$, and fractional shortening (FS) $(P=0.0011)$ (Supplemental Figure S1). These data add to an increasing body of literature supporting the effects of ONX-0914 in both counteracting the increase of first signs 

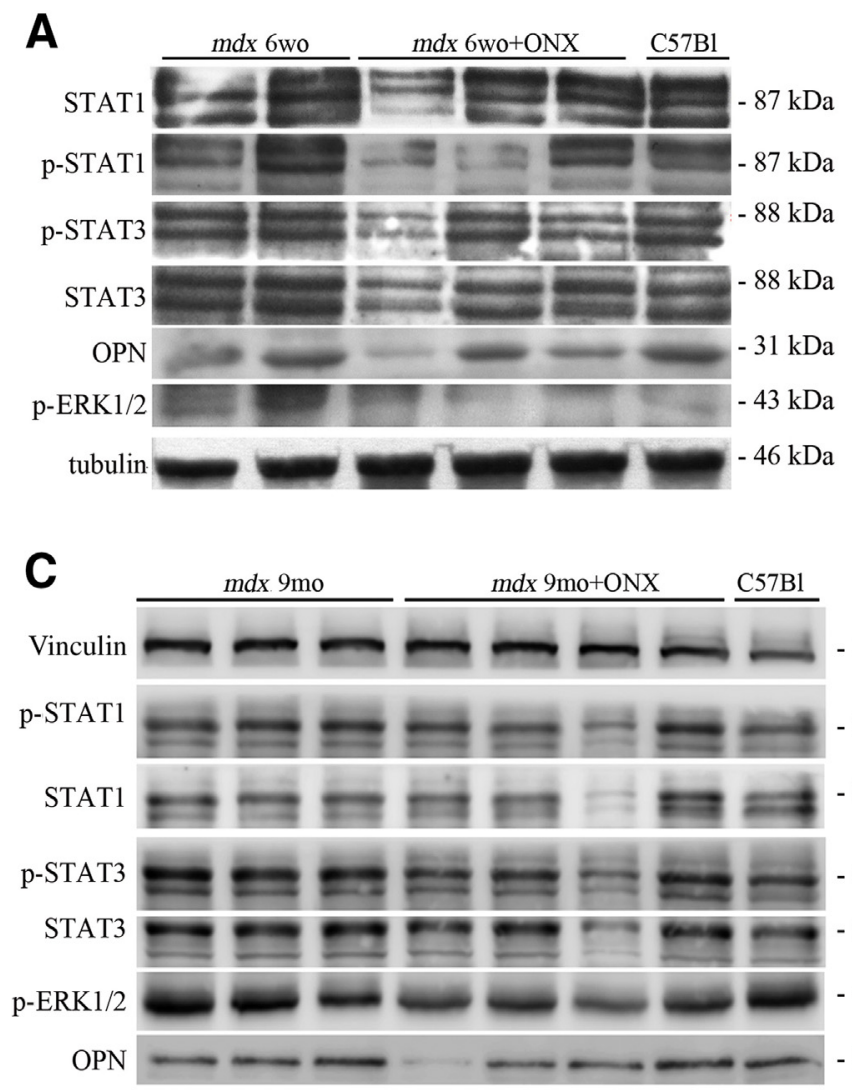

E
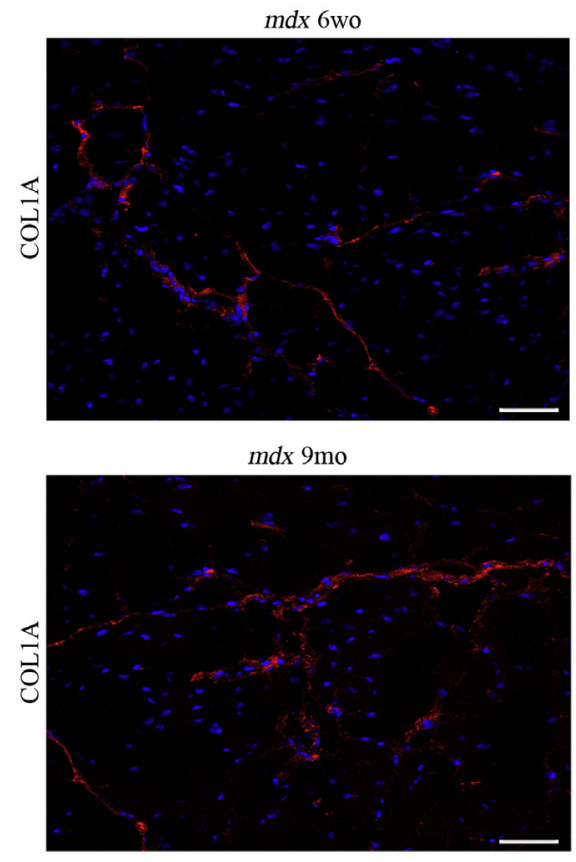

B
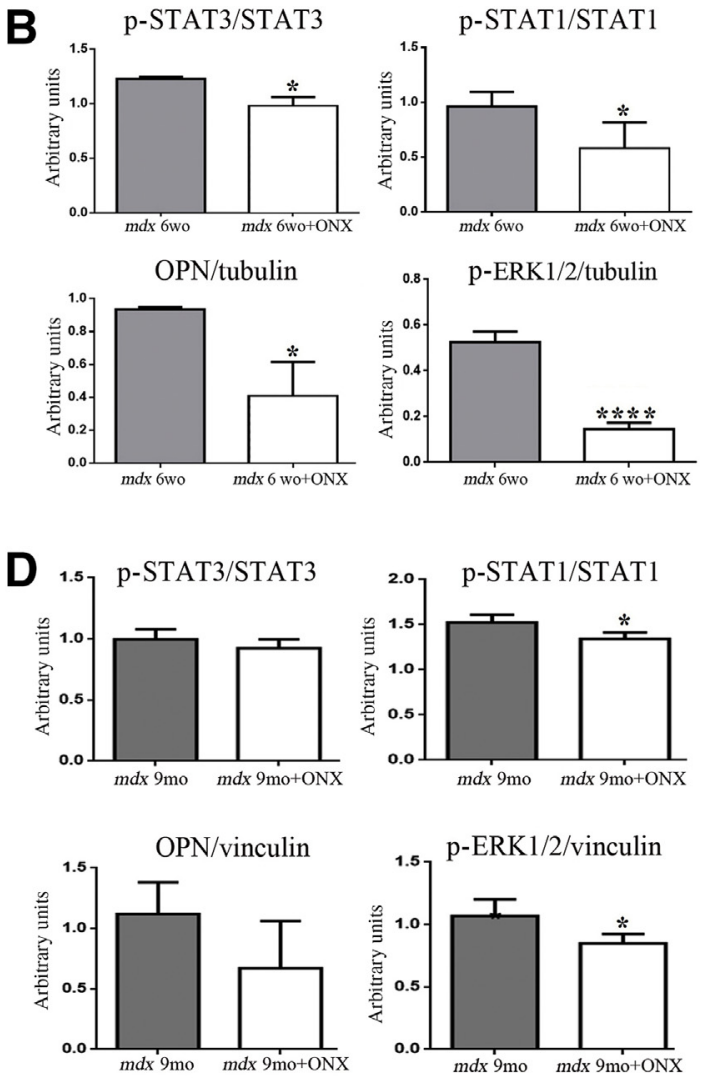

$-31 \mathrm{kDa}$

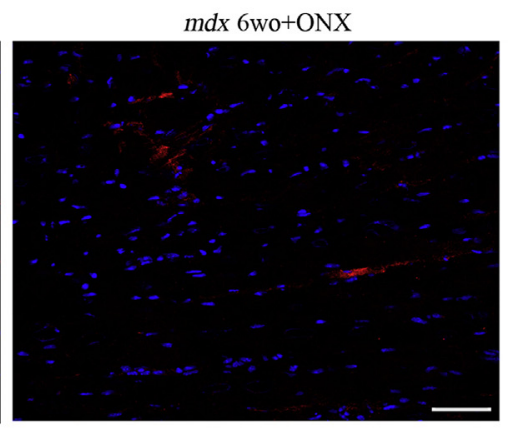

$\mathbf{F}$
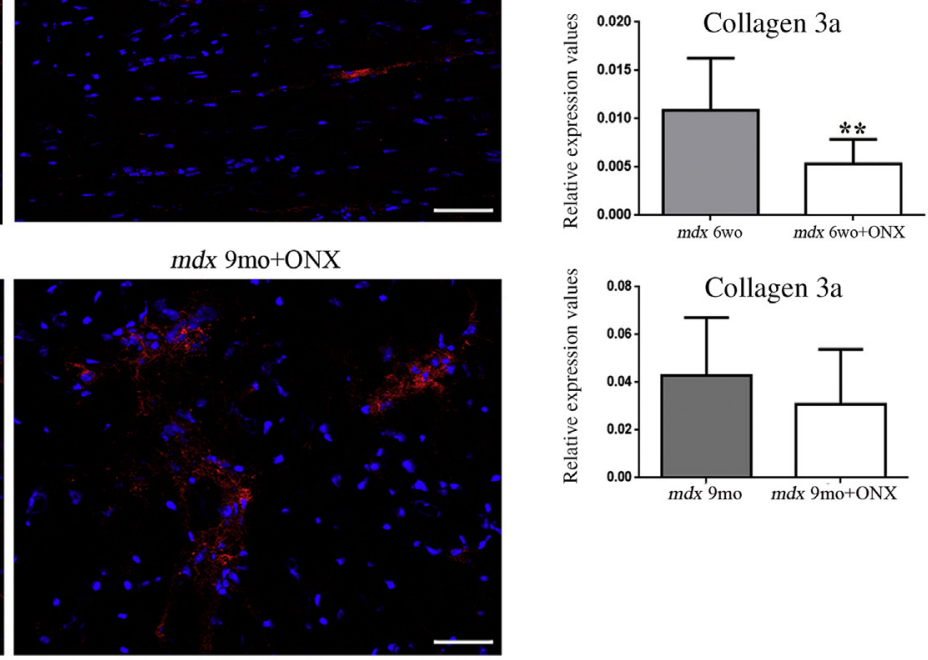

Figure 2 Amelioration of fibrotic mediators in $m d x$ mice after 0NX-0914 treatment. A: Measurement of the expression of pivotal profibrotic proteins in 6-week-old (6wo) $m d x$ mice by Western blotting analysis. B: Down-regulation of the phosphorylated STAT1 (p-STAT1)/STAT1 and p-STAT3/STAT3 ratios and osteopontin and phosphorylated ERK1/2 (p-ERK1/2) in 0NX-0914 treated 6wo $m d x$ mice. C and D: In 9-month-old (9mo) mdx mice a similar down-regulation of $\mathrm{p}$-ERK1/2 and p-STAT1/STAT1 ratio is seen, whereas osteopontin variation is not significant. E: Immunofluorescence staining reveals that 0 NX-0914 treatment determines the down-regulation of collagen 1a (COL1A) (in red) expression in both 6 wo and 9mo $m d x$ mice (representative images of the left ventricle). F: Quantitative RT-PCR experiments demonstrate a decrease in the COL3A gene transcript in 6 wo and 9 mo $m d x$ mice. Data are expressed as

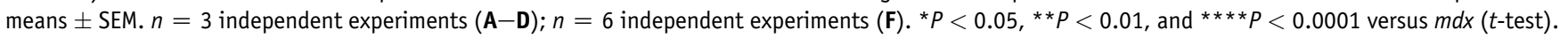
Scale bars $=50 \mu \mathrm{m}$. AU, arbitrary units. 
of cardiomyopathy in 6-week-old $m d x$ mice and in alleviating the development of the cardiomyopathy pathologic findings of 9-month-old $m d x$ mice.

\section{NX-0914 Treatment Diminishes Development of}

Fibrosis in 6-Week-Old and 9-Month-Old $m d x$ Mice

According to the well-known effects of ONX-0914 on inflammatory cell subpopulations, we hypothesized that our treatment could modify cardiac fibrosis and/or inflammation. Azan Mallory staining of the LV and right ventricle and of the interventricular septum revealed that 9-month-old $m d x$ mice were largely characterized by disorganized cardiomyocytes, prominent myocardial interstitial, and perivascular fibrosis, whereas 6-week-old $m d x$ mice had mild substantial myocardial deterioration and fibrotic deposition (Figure 1, E and F). The ONX-0914 treatment reduced significantly most signs of cardiac fibrosis in 6-week-old $(P=0.0180)$ (Figure 1E) and 9-month-old $(P=0.0023$ ) (Figure 1F) $m d x$ mice with comparatively mild perivascular fibrosis in the LV and interventricular septum and perivascular-restricted fibrous deposition in the right ventricle, thus agreeing with the functional benefits of ONX-0914 treatment for dystrophic myocardium.

Furthermore, the level of the mediator of fibrosis as osteopontin ( $P=0.0299$ and $P=0.1510$, respectively) (Figure 2, A-D) and phosphorylation of ERK1/2 $(P=0.0003$ and $P=0.0370$, respectively) (Figure $2, \mathrm{~A}-\mathrm{D}$ ) were significantly depressed in ONX-0914-treated hearts of 6-week-old and 9-month-old $m d x$ mice. Because osteopontin can interact
A
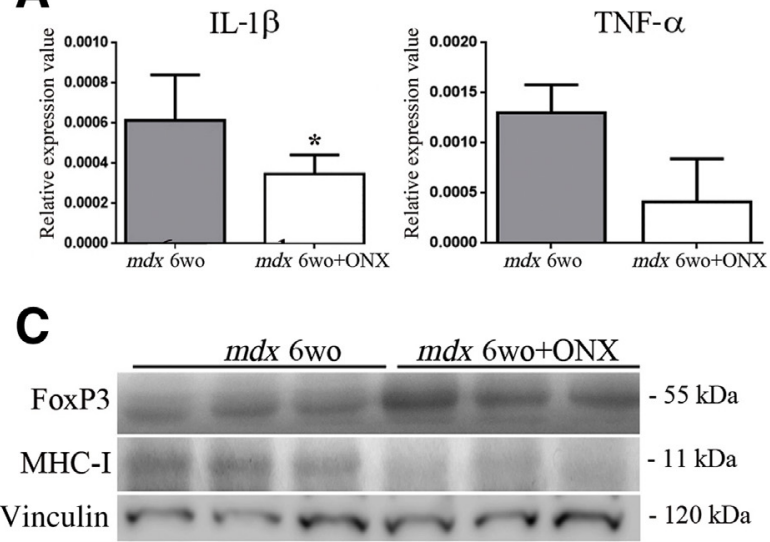

D
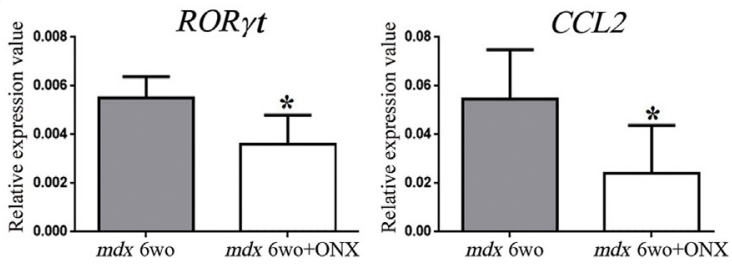

$\mathbf{F}$

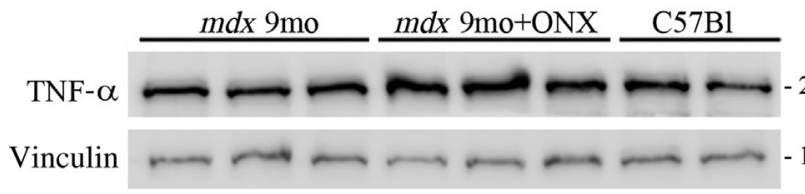

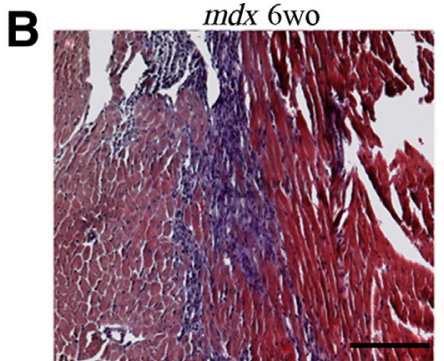
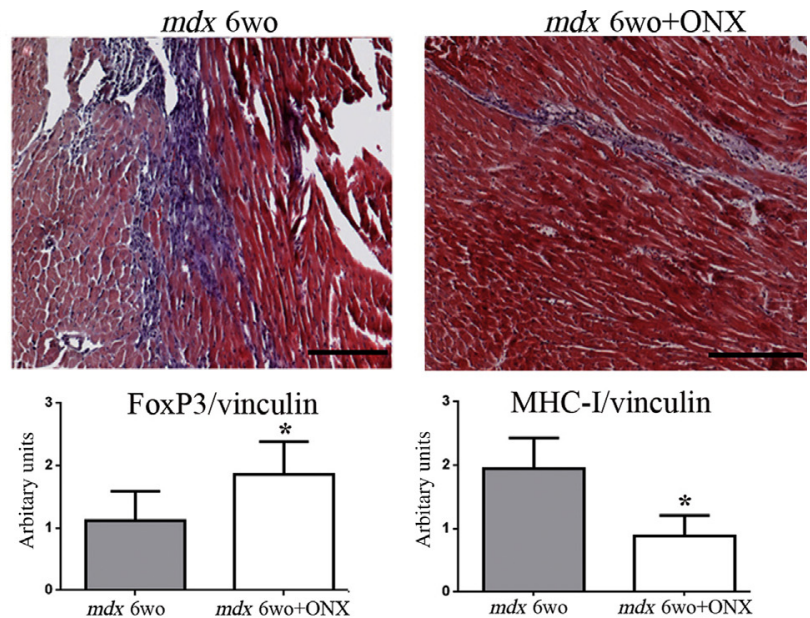

E
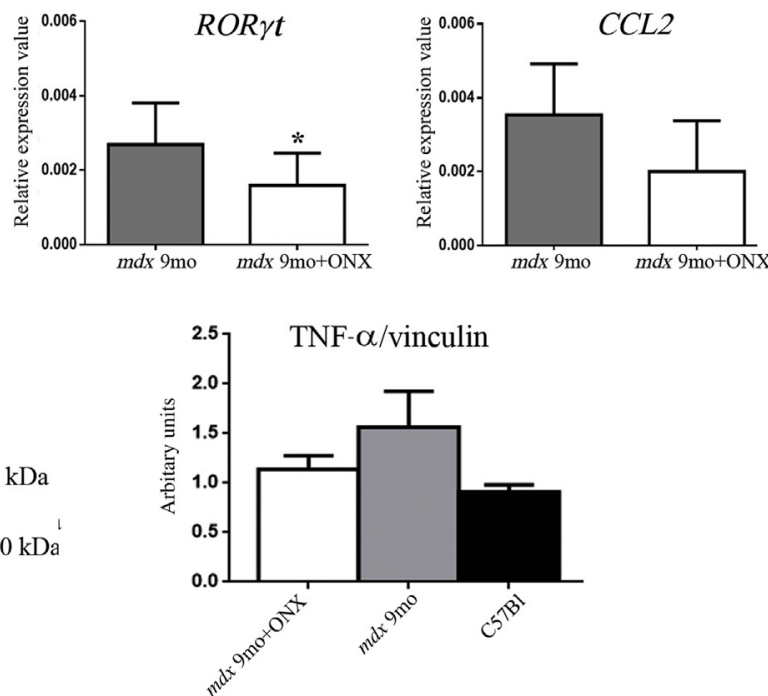

Figure 3 Immunoproteasome (IP) inhibition down-regulates inflammatory mediators in mdx mice. A: Quantitative RT-PCR experiments show a downregulation of the proinflammatory cytokines IL-1 $\beta$ and tumor necrosis factor $\alpha$ (TNF- $\alpha$ ) in 6 -week-old (6wo) mdx mice. B and C: Hematoxylin and eosin staining of 6wo $m d x$ heart tissue sections demonstrates the reduction of the infiltrating cells in the left ventricle of treated mice (B), whereas there is an overexpression of the FoxP3 protein and a slight but significant decrease in major histocompatibility complex I (MHC-I) (C). D: $R O R \gamma t$ and CCL2 expression is down-regulated in 0NX-0914-treated 6wo $m d x$ mice as assessed by quantitative RT-PCR experiments. E: In older $m d x$ mice, ROR $\gamma t$ expression is downregulated, whereas CCL2 amount is reduced but not significant. F: Western blotting analysis shows that TNF- $\alpha$ is down-regulated in 0NX-0914-treated 9-month-old (9mo) $m d x$ mice. Data are expressed as means \pm SEM. $n=6$ independent experiemnts $(\mathbf{A}) ; n=3$ independent experiments $(\mathbf{C}-\mathbf{E})$. ${ }^{\star} P<0.05$ versus $m d x$ (t-test). Scale bars $=200 \mu \mathrm{m}$. AU, arbitrary units; mo, months old; wo, weeks old. 

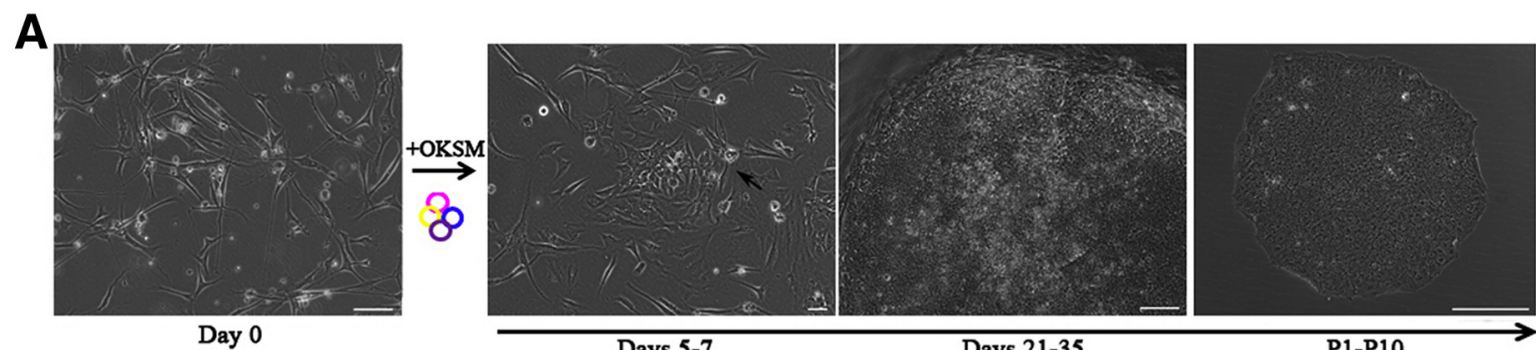

B

Alkaline phosphatase
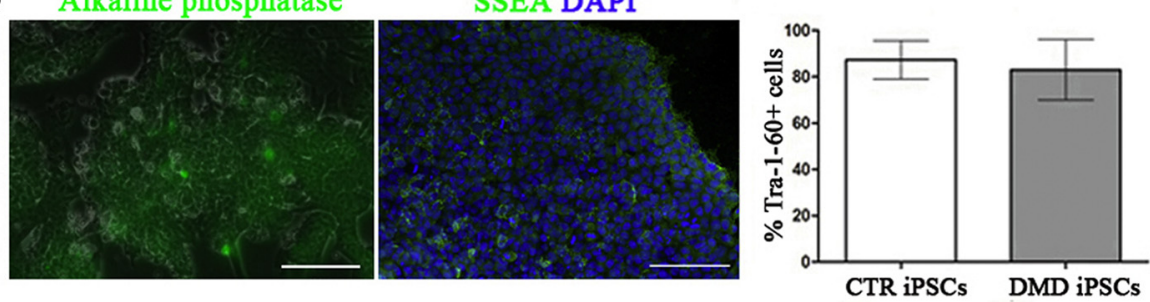

iPSCs
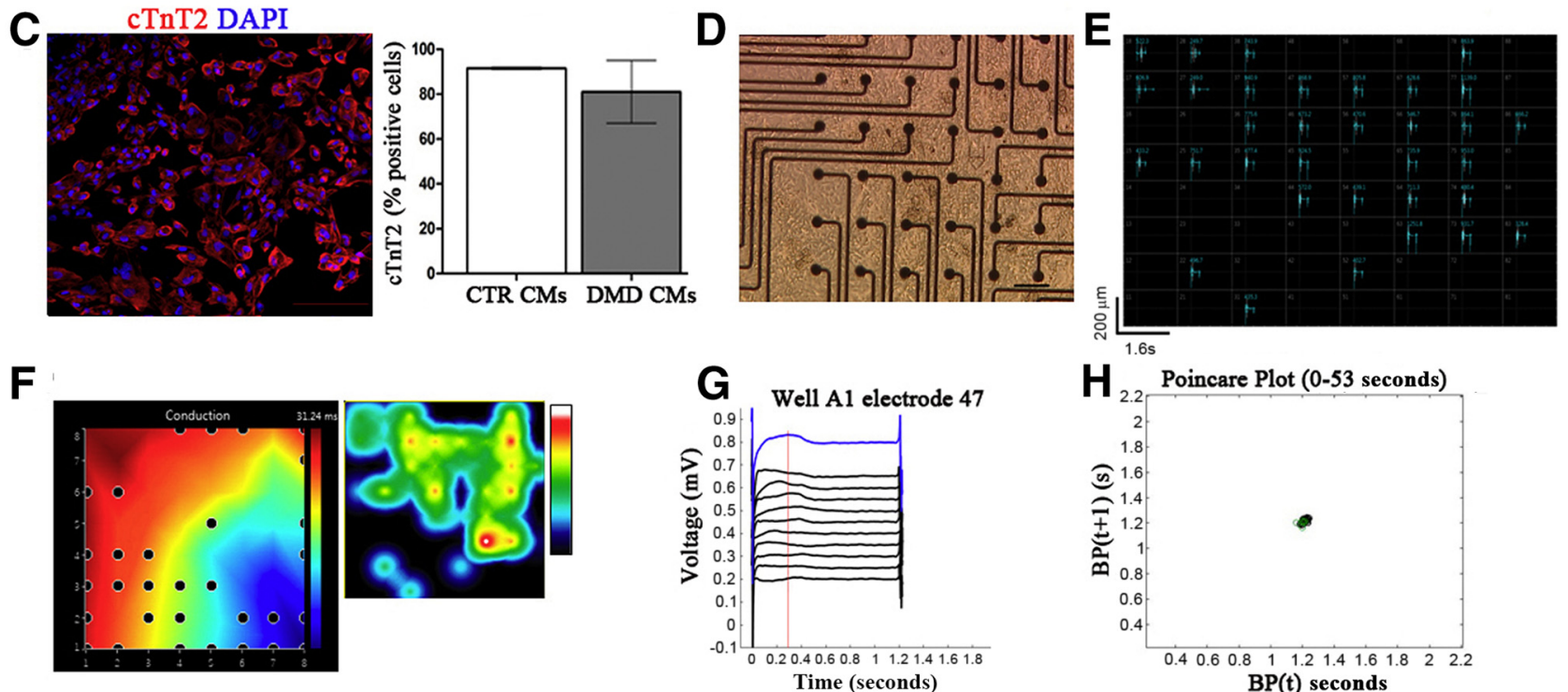

Figure 4 Derivation of cardiomyocytes from the induced pluripotent stem cells (iPSCs) of patients with Duchenne muscular dystrophy (DMD). A: Reprogramming of human dermal fibroblasts by transfection via electroporation with episomal vectors containing the Yamanaka factors. Arrow indicates the mesenchymal-to-epithelial transition phase of the reprogramming process. B: Immunohistochemical characterization of iPSCs for alkaline phosphatase activity and expression of stage-specific embryonic antigen-4 (SSEA4) and Tra-1-60 by immunohistochemistry and FACS analyses, respectively, in control (CTR) and DMD iPSC lines. C: Generation of high percentages of cardiac troponin T type $2-$ positive (cTnT2 ${ }^{+}$) cardiomyocytes derived from human iPSCs (CMs-d-iPSCs) from CTR and DMD iPSC lines through modulation of Wnt signaling in iPSCs. D: Replated CMs-d-iPSCs redevelop spontaneous beating 2 days after plating onto multielectrode array plates. E: Continuous voltage and conduction data were recorded for 2 minutes by the Maestro cardiac beat detector. F: Conduction propagation pattern of a well showing the spike arrival times of the initiation (blue) and termination (red) of a synchronized beat (black circles indicate that a beat was not detected on that electrode and $x$ and $y$ axis represent electrode column and row numbers). G: Beat waveforms from all electrodes were vertically aligned (black lines) followed by selection of the golden electrode [blue line, defined as an electrode with a repolarization traceable across all conditions and is the electrode used for field potential duration (FPD) analysis] for each well. Mean FPDs over the entire plate were calculated and plotted (red vertical line represents the golden channel T-wave peak). H: A Poincaré plot showed no discernible arrhythmic events (green circles indicate those beats used for calculating the mean beat waveform for the FDP detection). All analyses were performed using the Comprehensive in Vitro Proarrhythmia Assay analysis tool. Data are expressed as means \pm SEM. $n=3$ independent experiments (B); $n=4$ independent experiments (C). Scale bars: $100 \mu \mathrm{m}$ (A, Day 0); $50 \mu \mathrm{m}$ (A, Days 5-7); $100 \mu \mathrm{m}$ (A, Days 21-35); 200 $\mu \mathrm{m}$ (A, P1-P10); 100 $\mu \mathrm{m}$ $(B-D)$. $B P(t)$, beat period (seconds); $B P(t+1)$, previous beat period; OKSM, transient transcription factors; P1-P10, postoperative days 1 to 10.

with STAT proteins $^{29}$ and STAT-dependent pathways commonly regulate immunoproteasome structure and function, ${ }^{30-32}$ the expression of these proteins to uncover potential immunoproteasome-dependent molecular mechanisms regulating pathological fibrosis was investigated in $m d x$ mice with cardiomyopathy. After ONX-0914 treatment, the ratios between both total and phosphorylated STAT1 and STAT3 were significantly down-regulated $(P=0.0276$ and $P=0.0194$ for phosphorylated STAT3/STAT3 and phosphorylated STAT1/STAT1, respectively) (Figure 2, A and B). As in younger $m d x$ mice, treated 9-month-old immunoproteasome $m d x$ mice had a significant down-regulation of the 
ratio between both total and phosphorylated STAT1 $(P=0.0469)$ (Figure 2, C and D). Decreased levels of COL1A (Figure 2E) and collagen $3 \mathrm{a}(P=0.0085$ and $P=0.2477$ for 6-week-old and 9-month-old $m d x$ mice, respectively) (Figure 2F) found in ONX-0914-treated hearts of $m d x$ mice agree with the observed differences in fibrosis.

\section{NX-0914 Down-Regulated the Inflammatory}

Responses in 6-Week-0ld and 9-Month-0ld mdx Mice

Accordingly, with modifications in the extent of fibrosis, the amount of proinflammatory cytokines expressed in cardiac samples from treated and untreated 6-week-old $m d x$ mice were determined: quantitative RT-PCR experiments proved that the proinflammatory cytokines IL-1 $\beta$ and TNF- $\alpha$ were overexpressed in 6-week-old untreated $m d x$ mice compared with ONX-0914-treated ones $(P=0.0250$ for IL-1 $\beta$ and $P=0.151$ for TNF- $\alpha$ ) (Figure 3A). Thus, the amount of immune infiltrating cells that normally secrete proinflammatory IL- $1 \beta$ and TNF- $\alpha$ were evaluated, and significant reduction of these cells (ranging from 10 to $>100$ cells) was found in the hearts of ONX-0914-treated 6-week-old $m d x$ mice (Figure 3B). Interestingly, the $\mathrm{CD}^{+} \mathrm{CD} 45^{+}$ subpopulation (B and $\mathrm{T}$ lymphocytes) was significantly decreased in ONX-0914-treated hearts of 6-week-old $m d x$ mice $(P<0.01)$, whereas no differences in the percentage of $\mathrm{CD}_{107^{+}}$macrophages was found (data not shown). The specific reduction of $\mathrm{B}$ and $\mathrm{T}$ lymphocytes on immunoproteasome inhibition was corroborated by detection of significantly reduced major histocompatibility complex I expression $(P=0.0387)$ and overexpression of FoxP3, the specific marker of T-regulatory cells that act to suppress immune response $(P=0.0357$ ) (Figure $3 \mathrm{C}$ ). In addition, quantitative RT-PCR experiments revealed that ONX-0914 treatment reduced the expression of retinoic acid receptor-related orphan receptor $\gamma(\mathrm{ROR}-\gamma \mathrm{t})$, a master regulator of proinflammatory Th17 lymphocytes $(P=0.0472)$ and macrophagespecific marker chemokine (C-C motif) ligand 2 (CCL2) $(P=0.0212)$ in hearts of 6-week-old $m d x$ mice (Figure $3 \mathrm{D})$.

Similar to pathologic features described in 6-week-old $m d x$ mice, in 9-month-old $m d x$ mice a down-regulation of ROR- $\gamma \mathrm{t}$ $(P=0.0414)$ and CCL2 (Figure 3E) was seen, and ONX0914 treatment diminished the amount of TNF- $\alpha$ (Figure 3F). This evidence suggests that immune cell and cytokine reduction was significant at the onset of detectible cardiac functional decline, whereas reduced fibrosis, improved
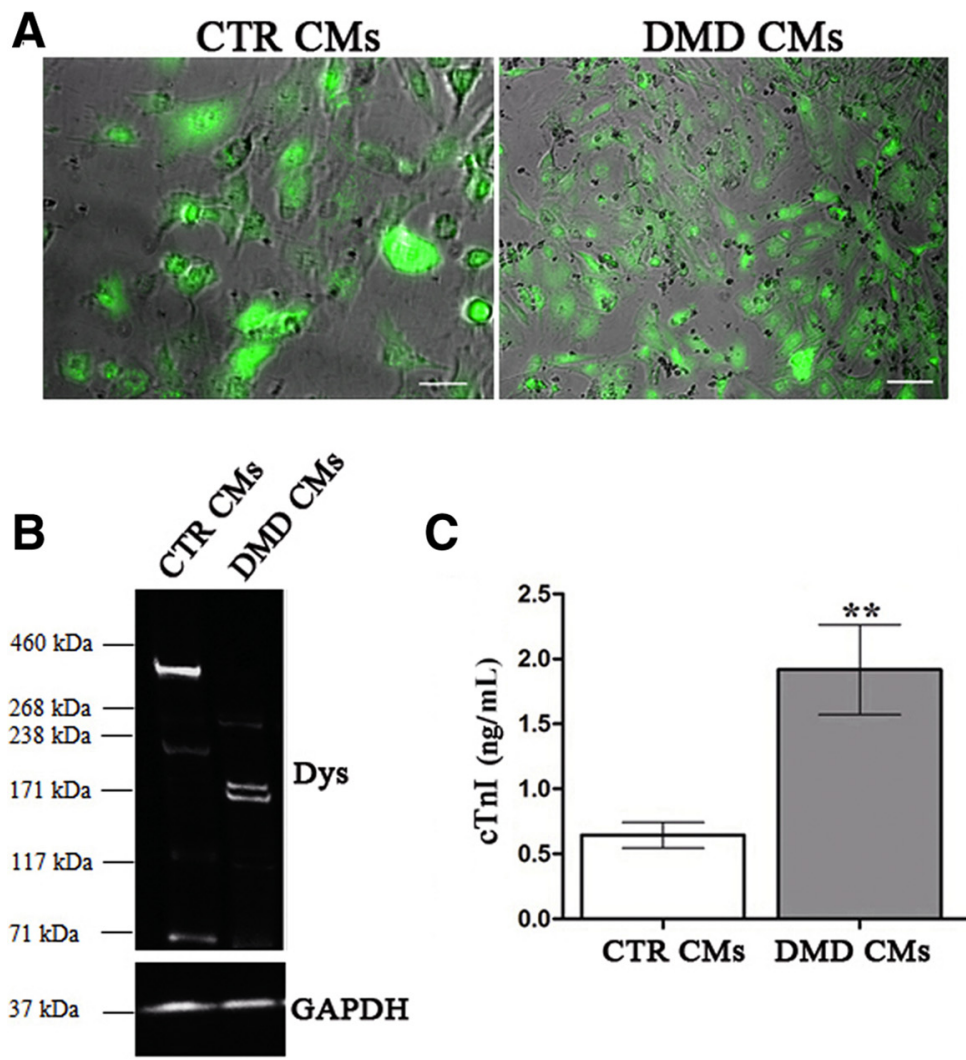

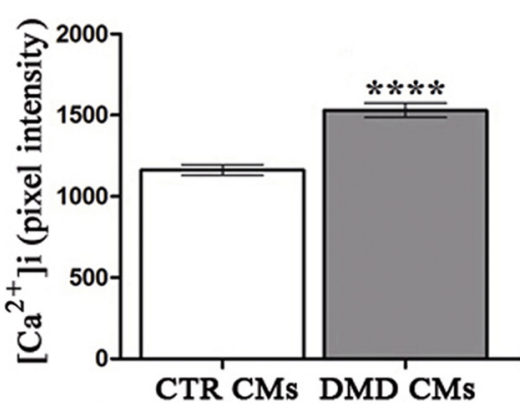

C
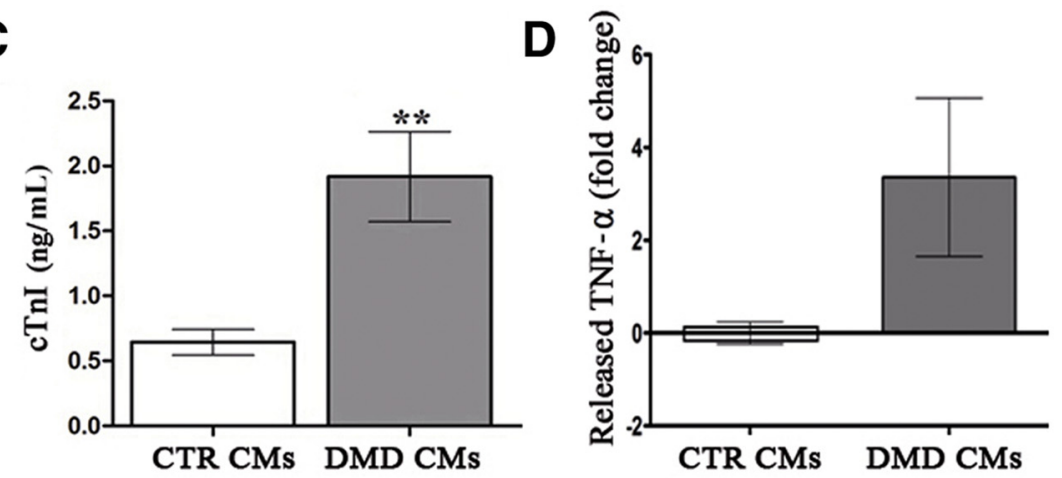

Figure 5 Characterization of muscular dystrophy cardiomyopathy hallmarks in patient-specific cardiomyocytes (CMs) derived from human induced pluripotent stem cells (iPSCs) (CMs-d-iPSCs). A: Representative staining of control (CTR) and Duchenne muscular dystrophy (DMD) CMs with the green fluorescent calcium indicator Flou-4 AM (green) shows increased intracellular $\mathrm{Ca}^{2+}\left[\left(\mathrm{Ca}^{2+}\right) \mathrm{i}\right]$ in DMD versus CTR CMs-d-iPSCs, as indicated by the histogram obtained by calculating the intensity of pixels. B: As expected, expression of full-length dystrophin (427 kDa) was absent in DMD compared with CTR CMs-diPSCs. C and D: Histograms reveal that DMD CMs-d-iPSCs release significantly higher amounts of cardiac troponin I (cTnI) (C) and tumor necrosis factor $\alpha$ (TNFa) (D) compared with CTR CMs-d-iPSCs. Data are expressed as means \pm SEM. $n=5$ to 8 independent experiments $(\mathbf{A}$ and $\mathbf{B}) ; n=6$ to 7 independent experiments (C and $\mathbf{D}) .{ }^{* *} P<0.01,{ }^{* * * *} P<0.0001$ versus CTR CMs ( $t$-test). Scale bars $=100 \mu \mathrm{m}$. 

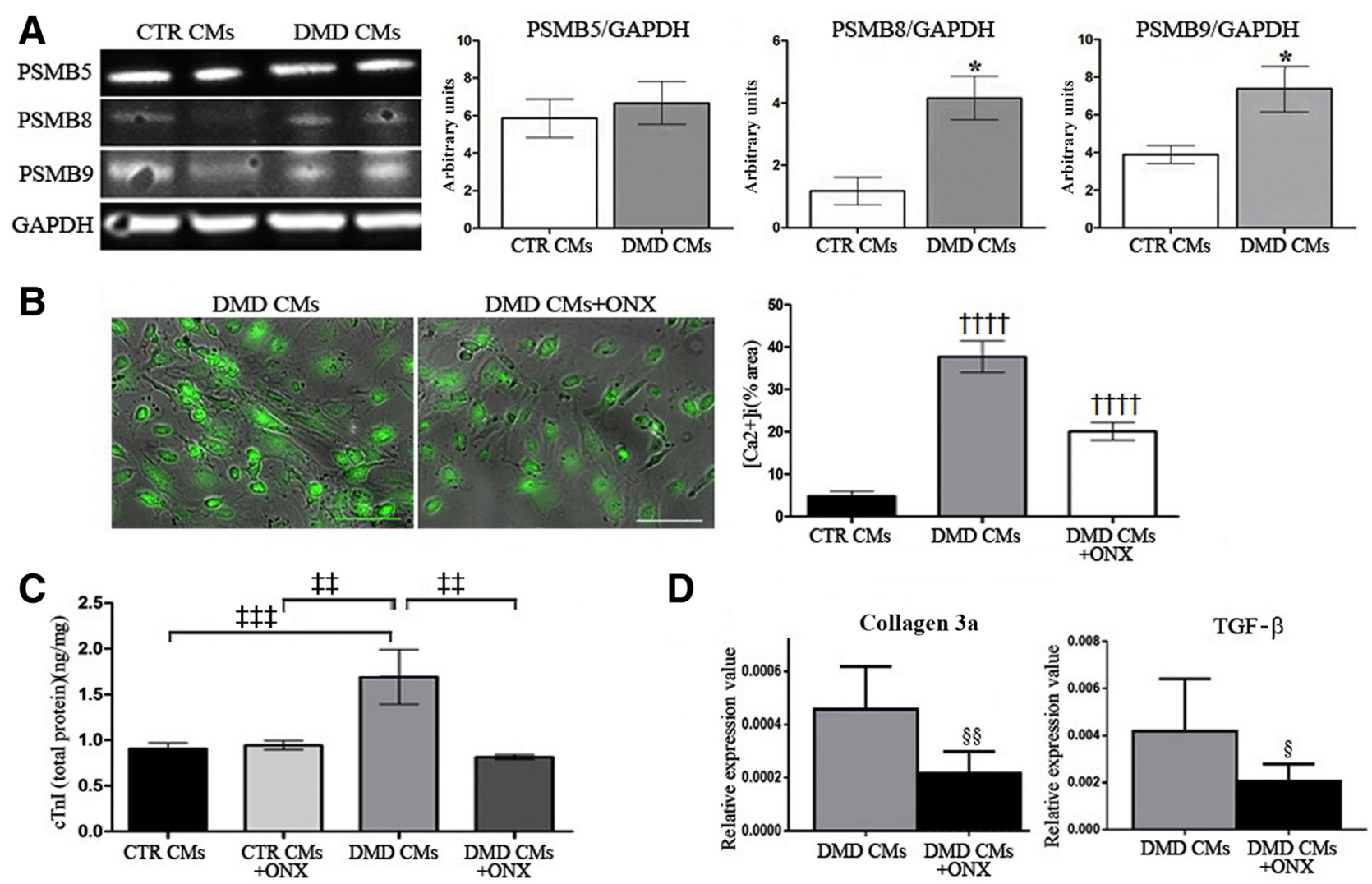

Figure 6 Expression of immunoproteasome (IP) in control (CTR) and Duchenne muscular dystrophy (DMD) cardiomyocytes (CMs) derived from human induced pluripotent stem cells (CMs-d-iPSCs) and the effect of IP inhibition. Measurement of IP expression in CTR and DMD CMs-d-iPSCs. A: Western blotting analysis reveals that DMD CMs-d-iPSCs up-regulate the IP subunits PSMB8 and PSMB9; however, the expression of PSMB5 is unchanged. B and C: Representative staining of CTR and DMD cardiomyocytes with the green fluorescent calcium indicator Fluo-4 AM (green). Treatment of CTR and DMD CMs-d-iPSCs for 7 days (from day 9 after CM differentiation) reverses the increases in intracellular $\mathrm{Ca}^{2+}\left[\left(\mathrm{Ca}^{2+}\right) \mathrm{i}\right](\mathbf{B})$ and reduces the release of cardiac troponin I (cTnI), meaning that the effect of IP inhibition on lowering CTnI levels is significantly greater for DMD CMs compared with CTR CMs (C). D: Quantitative RT-PCR experiments confirm the down-regulation of collagen $3 a$ and transforming growth factor $\beta$ (TGF- $\beta$ ) in treated DMD CMs-d-iPSCs. Data are expressed as means \pm SEM. $n=4$ to 17 independent experiments (A); $n=9$ to 11 independent experiments (B); $n=3$ to 18 independent experiments (C); $n=8$ independent experiments (D). ${ }^{*} P<0.05$ versus CTR CMs (t-test); ${ }^{\dagger \dagger \dagger \dagger} P<0.0001$ versus CTR CMs (one-way analysis of variance); ${ }^{\ddagger \ddagger} P<0.01,{ }^{\ddagger \ddagger \ddagger} P<0.001$ (twoway analysis of variance); ${ }^{\S} P<0.05$, and ${ }^{\S \S} P<0.01$ versus DMD CMs (t-test). Scale bars $=100 \mu \mathrm{m}$.

diastolic filling, and amelioration of cardiac output were equally present in both early and advanced cardiac decline.

\section{ONX-0914 Affects Cardiac Mitochondrial Functions Only in 9-Month-Old $m d x$ Mice}

According to several works, DMD tissues have a deficit in resting ATP levels ${ }^{33}$; consequently, it was reported that active mitochondria were necessary to rescue sarcolemmal abnormalities in myofibers. ${ }^{34}$ To determine whether the diminished fibrosis was partially attributable to an amelioration of the amount and functionality of mitochondria, several mitochondrial enzymes were studied in the hearts of ONX-treated and untreated 6-week-old and 9-month-old $m d x$ mice. As expected, two-way analysis of variance revealed increased mitochondrial activity in aged $m d x$ mice with significant upregulation of NADH dehydrogenase $(P=0.0140)$, NADH cytochrome $c$ reductase $(P=0.008)$, succinate cytochrome $c$ reductase $(P=0.0007)$, and cytochrome oxidase $(P<0.0001)$ (Supplemental Figure S2A). Conversely, ONX-0914 treatment of 9-month-old $m d x$ mice determined significant reduction of the enzymatic activity of NADH cytochrome $c$ reductase $(P=0.0337)$ and, in particular, of succinate cytochrome $c$ reductase $(P=0.0009)$ and cytochrome oxidase $(P<0.0001)$ (Supplemental Figure S2A), thus resembling the values of 6-week-old $m d x$ mice. Because the abnormal cytosolic $\mathrm{Ca}^{2+}$ levels present in $m d x$ cardiomyocytes led to an age-dependent degeneration of mitochondrial structure and function, ${ }^{35}$ the expression TRPC1 was evaluated for calcium. Although TRPC1 is up-regulated in the hearts of $m d x$ mice, confirming previous evidence, ${ }^{36}$ no differences were found between ONX-0914-treated and untreated 6-week-old and 9-month-old $m d x$ mice (Supplemental Figure S2B).

\section{Cardiomyocytes Derived from Dystrophic iPSCs}

Recapitulate Abnormal Immunoproteasome Expression as Observed in $m d x$ Mice

It is extremely difficult to obtain cardiac biopsy specimens from patients with muscular dystrophy, therefore, to confirm the interesting results derived from $m d x$ mice, human 
dermal fibroblasts were reprogrammed with nonintegrating episomal vectors containing the Yamanaka factors ${ }^{37}$ (Figure 4A). Several lines of iPSCs were established from healthy controls and patients with DMD under feeder-free defined conditions, ${ }^{38}$ which had pluripotent morphologic findings and expressed alkaline phosphatase, SSEA4, and Tra-1-60, as assessed by immunofluorescence and FACS $(87 \pm 8$ versus $83 \% \pm 13 \%$ Tra-1-60 positive cells $)$ (Figure 4B). Cardiomyocytes were derived from control and DMD iPSC lines (CMs-d-iPSCs) through the temporal modulation of Wnt signaling in iPSCs grown in defined feeder-free monolayers as described elsewhere. ${ }^{24}$ No significant difference was found in the percentage of cTnT2positive cells $\left(92 \pm 1\right.$ versus $81 \% \pm 14 \%$ cTnT2 $^{+}$cells) (Figure 4C). CMs-d-iPSCs had spontaneous beating with electrical activity similar to other reports in the literature 39,40 (Figure 4, D-G). Interestingly, through Poincare Plot the absence of unstable or abnormal beats as in arrhythmias was also determined (Figure 4H). CMs-d-iPSCs were maintained as beating monolayers until day 16 of cardiomyocyte differentiation followed by the assessment of DMD cardiac disease hallmarks. An up-regulation of intracellular $\mathrm{Ca}^{2+}$ in DMD CMs-d-iPSCs but not in CTR CMs-d-iPSCs was observed $(1162 \pm 33$ versus $1530 \pm 45$ mean pixel intensity, $P<0.0001$ ), which correlates with our previous observation of increased function of voltage-gated calcium channels association with DMD and cardiomyopathy pathophysiologic alterations (Figure 5A). ${ }^{41}$ In DMD CMs-diPSCs, full-length dystrophin protein was absent compared with control CMs-d-iPSCs (Figure 5B). Furthermore, DMD CMs-d-iPSCs recapitulated relevant clinical characteristics of DMD because they displayed increased release of the cardiac damage marker cTnI $(0.65 \pm 0.10$ versus $1.92 \pm 0.35 \mathrm{ng}$ of cTnI per milligram of total protein, $P=0.0072)$ (Figure 5C) and of TNF- $\alpha(-0.8128 \pm 0.2386$ versus $3.358 \pm 1.705$ ) (Figure $5 \mathrm{D}$ ). The expression of constitutive and immunoproteasome subunits in the CMs-diPSCs was determined. The PSMB5 subunit remained unaltered in DMD CMs-d-iPSCs; the immunoproteasome subunits PSMB8 $(P=0.0417)$ and PSMB9 $(P=0.0377)$ were significantly overexpressed in DMD CMs-d-iPSCs compared with control CMs-d-iPSCs (Figure 6A). Next, the effect of ONX-0914 treatment was monitored on cardiomyopathy hallmarks. Significantly, immunoproteasome inhibition dampened intracellular $\mathrm{Ca}^{2+}(P<0.0001$ for both DMD CMs and DMD CMs + ONX-0914 versus control CMs; 1-way analysis of variance) (Figure 6B) and reduced the release of cTnI related to DMD CMs (with $P=0.0013, P=0.0094$, and $P=0.0399$ for control CMs, control CMs + ONX, and DMD CMs + ONX, respectively; two-way analysis of variance) (Figure 6C). Interestingly, quantitative RT-PCR found the down-regulation of transforming growth factor $\beta$ and collagen $3 \mathrm{a}$ expression in treated CMs-d-iPSCs $(P=0.0235$ and $P=0.0020$, respectively) (Figure 6D). Control and DMD CMs-d-iPSCs treated with $200 \mathrm{nmol} / \mathrm{L}$ of ONX-0914 had a severe acute cardiotoxic response, which was not observed at concentrations $<150 \mathrm{nmol} / \mathrm{L}$, demonstrating a dose-dependent toxic pattern in CMs-d-iPSCs. Specifically, 2 days after treatment, CMs-d-iPSCs started displaying abnormal behavior and were on visual inspection of a distinct poorer quality to vehicle-treated CMs-d-iPSCs. After 3 to 4 days of treatment, most CMs-d-iPSCs stopped contracting and displayed a further deterioration in cellular morphologic features. After 7 days of treatment, most CMs-d-iPSCs were detached and necrotic (Supplemental Figure S3A), and the few remaining CMs-d-iPSCs had overt aberrations in morphologic findings (Supplemental Figure S3, B-D).

\section{Discussion}

Dilated cardiomyopathy is the major cause of death of patients with DMD and is predominantly characterized by severe inflammation and fibrosis. Unfortunately, dilated cardiomyopathy is unresponsive to usual care based on glucocorticoids, ${ }^{42,43}$ increasing the urgencies for novel treatments aimed at improving the poor DMD clinical outcomes and preventing fatal heart failure. The murine model of DMD, the $m d x$ mouse, develops dilated cardiomyopathy by 32 weeks of age, when accumulation of connective tissue and contractile deviances is undeniable. ${ }^{44}$ However, contractile dysfunctions and development of ventricular inflammation and fibrosis have already been described in $m d x$ mice as early as 2 months of age. ${ }^{6,7}$ Immunoproteasome functions are synergistically associated with the activity of most of the proteins that are involved in the development of the symptoms that will lead to dilated cardiomyopathy, such as cardiomyocyte necrosis, myocardial tissue degeneration, and fibrosis. ${ }^{22,45,46} \mathrm{We}$ found overexpression of immunoproteasome subunits in the hearts of $m d x$ mice and suggest that immunoproteasome modulation is a valuable tool to counteract cardiac pathologic findings in $m d x$ mice.

The immunoproteasome inhibition by ONX-0914 treatment decreased inflammatory cell activation, proinflammatory cytokine expression, and fibrosis and ameliorated the hemodynamic performance of $m d x$ mice with early ( 6 weeks old) and advanced ( 9 months old) dilated cardiomyopathy. Interestingly, immunoproteasome modulation determined the delay of cardiomyopathy in $m d x$ mice with early dilated cardiomyopathy (6 weeks old) and improved the cardiac performance of older $m d x$ mice with advanced dilated cardiomyopathy (9 months old). LV dysfunction was prominent in untreated 9-month-old $m d x$ mice. ONX-0914 administration before the onset of cardiac functional decline preserved LV systolic function (ie, ejection fraction and wall thickness). Improvements in the histologic findings in the hearts of $m d x$ mice resulted from the treatment correlated well with the functional improvements detected by echocardiography. Outstandingly, inhibition of PSMB8 in 6-week-old $m d x$ mice 
down-regulated the expression of phosphorylated ERK1/2 that potentially phosphorylate STATs and thus control collagen expression (whose deposition is typical of myocardial fibrosis). Importantly, ONX-0914 treatment reduced the percentage of infiltrating $\mathrm{B}$ and $\mathrm{T}$ cells in the hearts of $m d x$ mice, which in turn cause the downregulation of NF- $\kappa \mathrm{B}$, hampering its cooperation with STATs. Such effects may also be attributed to reduced expression of inflammatory cytokines, such as ROR- $\gamma \mathrm{t}$, IL$1 \beta$, and TNF- $\alpha$, that activate the JAK/STAT pathways. Interestingly, a down-regulation of the fibrotic enhancer, osteopontin, and decreased levels of collagen 1a and 3a, which agree with the observed differences in fibrosis of ONX-0914-treated $m d x$ mice, were observed. These data are in accordance with those published by other groups reporting the immunomodulatory role of osteopontin in $\mathrm{DMD}^{47}$ and its association with matrix metalloproteinase 9. ${ }^{48}$ Recently, in a cohort of 40 patients with DMD and early myocardial disease, Raman et $\mathrm{al}^{49}$ found a combinatorial effect of angiotensin-converting enzyme inhibitor and angiotensin receptor blocker with the drug eplerenone (commonly used in the management of chronic heart failure), which improved cardiac features possibly through a modulation of an osteopontin-dependent pathway. Thus, we propose osteopontin as a reliable target to ameliorate cardiac fibrosis in patients with DMD. ${ }^{50}$ Nevertheless, the reduced inflammatory activation and fibrosis obtained in ONX-0914-treated hearts of $m d x$ mice lead to improved hemodynamic performance characterized by increased LV ejection fraction and decreased LV end-systolic and end-diastolic volumes. Because substantial improvement of dilated cardiomyopathy was observed in ONX0914-treated $m d x$ mice, it was analyzed whether the same effect could be translated to DMD. Thus, dermal fibroblasts isolated from patients with DMD and healthy individuals were reprogrammed to iPSCs, which were then differentiated to functional beating CMs-d-iPSCs. It was confirmed that DMD CMs-d-iPSCs overexpressed the immunoproteasome subunits and their inhibition protected cardiomyocytes from damage as indicated by decreased release of cTnI and TNF- $\alpha$. In addition, the antifibrotic function of ONX-0914-at a nontoxic concentration of $150 \mathrm{nmol} / \mathrm{L}$ because cardiotoxicity was determined when ONX-0914 was used at $200 \mathrm{nmol} / \mathrm{L}$ - could be exerted through down-regulation of transforming growth factor $\beta$ and collagen 1a expression. Interestingly, in an animal model of hypertension, the overexpression of the immunoproteasome subunit PSMB10 was found to be responsible for increasing atrial fibrosis through the TGF- $\beta$ and SMAD2/3 pathways and for the up-regulation of inflammation and oxidative stress mediated by NF- $\kappa \mathrm{B} .{ }^{18}$ Taken together, these data reveal the beneficial effects of ONX0914 to treat and prevent dilated cardiomyopathy in $m d x$ mice, ranking the immunoproteasome inhibitor compounds among the new drugs to be tested in future DMD clinical trials. ${ }^{51,52}$

\section{Acknowledgments}

We thank the Associazione Amici del Centro Dino Ferrari. Y.T., G.P., A.F., C.S., and A.G. conceived and designed the experiments; C.S., A.F., and A.G. wrote the paper; A.F., A.G., P.N., and G.P.C. interpreted and analyzed the data; G.M., E.C., D.R., A.S., P.B., and F.F. performed the experiments.

\section{Supplemental Data}

Supplemental material for this article can be found at https://doi.org/10.1016/j.ajpath.2018.10.010.

\section{References}

1. Beynon RP, Ray SG: Cardiac involvement in muscular dystrophies. QJM 2008, 101:337-344

2. Judge DP, Kass DA, Thompson WR, Wagner KR: Pathophysiology and therapy of cardiac dysfunction in Duchenne muscular dystrophy. Am J Cardiovasc Drugs 2011, 11:287-294

3. Verhaert D, Richards K, Rafael-Fortney JA, Raman SV: Cardiac involvement in patients with muscular dystrophies: magnetic resonance imaging phenotype and genotypic considerations. Circ Cardiovasc Imaging 2011, 4:67-76

4. Kamdar F, Garry DJ: Dystrophin-deficient cardiomyopathy. J Am Coll Cardiol 2016, 67:2533-2546

5. Williams IA, Allen DG: Intracellular calcium handling in ventricular myocytes from mdx mice. Am J Physiol Heart Circ Physiol 2007, 292:H846-H855

6. Danialou G, Comtois AS, Dudley R, Karpati G, Vincent G, Des Rosiers C, Petrof BJ: Dystrophin-deficient cardiomyocytes are abnormally vulnerable to mechanical stress-induced contractile failure and injury. FASEB J 2001, 15:1655-1657

7. Nakamura A, Yoshida K, Takeda S, Dohi N, Ikeda S: Progression of dystrophic features and activation of mitogen-activated protein kinases and calcineurin by physical exercise, in hearts of mdx mice. FEBS Lett 2002, 520:18-24

8. Quinlan JG, Hahn HS, Wong BL, Lorenz JN, Wenisch AS, Levin LS: Evolution of the mdx mouse cardiomyopathy: physiological and morphological findings. Neuromuscul Disord 2004, 14:491-496

9. Barry SP, Townsend PA, Latchman DS, Stephanou A: Role of the JAK-STAT pathway in myocardial injury. Trends Mol Med 2007, 13: $82-89$

10. Xuan YT, Guo Y, Han H, Zhu Y, Bolli R: An essential role of the JAK-STAT pathway in ischemic preconditioning. Proc Natl Acad Sci U S A 2001, 98:9050-9055

11. Hilfiker-Kleiner D, Hilfiker A, Fuchs M, Kaminski K, Schaefer A, Schieffer B, Hillmer A, Schmiedl A, Ding Z, Podewski E, Podewski E, Poli V, Schneider MD, Schulz R, Park JK, Wollert KC, Drexler H: Signal transducer and activator of transcription 3 is required for myocardial capillary growth, control of interstitial matrix deposition, and heart protection from ischemic injury. Circ Res 2004, 95:187-195

12. Dai B, Cui M, Zhu M, Su WL, Qiu MC, Zhang H: STAT1/3 and ERK1/2 synergistically regulate cardiac fibrosis induced by high glucose. Cell Physiol Biochem 2013, 32:960-971

13. Ebstein F, Kloetzel PM, Kruger E, Seifert U: Emerging roles of immunoproteasomes beyond $\mathrm{MHC}$ class I antigen processing. Cell Mol Life Sci 2012, 69:2543-2558

14. Chen CN, Graber TG, Bratten WM, Ferrington DA, Thompson LV Immunoproteasome in animal models of Duchenne muscular dystrophy. J Muscle Res Cell Motil 2014, 35:191-201 
15. Zu L, Bedja D, Fox-Talbot K, Gabrielson KL, Van Kaer L, Becker LC, Cai ZP: Evidence for a role of immunoproteasomes in regulating cardiac muscle mass in diabetic mice. J Mol Cell Cardiol 2010, 49:5-15

16. Opitz E, Koch A, Klingel K, Schmidt F, Prokop S, Rahnefeld A, Sauter M, Heppner FL, Volker U, Kandolf R, Kuckelkorn U, Stangl K, Kruger E, Kloetzel PM, Voigt A: Impairment of immunoproteasome function by beta5i/LMP7 subunit deficiency results in severe enterovirus myocarditis. PLoS Pathog 2011, 7:e1002233

17. Cosper PF, Harvey PA, Leinwand LA: Interferon-gamma causes cardiac myocyte atrophy via selective degradation of myosin heavy chain in a model of chronic myocarditis. Am J Pathol 2012, 181: 2038-2046

18. Li J, Wang S, Bai J, Yang XL, Zhang YL, Che YL, Li HH, Yang YZ: Novel role for the immunoproteasome subunit PSMB10 in angiotensin II-induced atrial fibrillation in mice. Hypertension 2018, 71: $866-876$

19. Basler M, Mundt S, Bitzer A, Schmidt C, Groettrup M: The immunoproteasome: a novel drug target for autoimmune diseases. Clin Exp Rheumatol 2015, 33:S74-S79

20. Mundt S, Basler M, Buerger S, Engler H, Groettrup M: Inhibiting the immunoproteasome exacerbates the pathogenesis of systemic Candida albicans infection in mice. Sci Rep 2016, 6:19434

21. Muchamuel T, Basler M, Aujay MA, Suzuki E, Kalim KW, Lauer C, Sylvain C, Ring ER, Shields J, Jiang J, Shwonek P, Parlati F, Demo SD, Bennett MK, Kirk CJ, Groettrup M: A selective inhibitor of the immunoproteasome subunit LMP7 blocks cytokine production and attenuates progression of experimental arthritis. Nat Med 2009, 15:781-787

22. Althof N, Goetzke CC, Kespohl M, Voss K, Heuser A, Pinkert S, Kaya Z, Klingel K, Beling A: The immunoproteasome-specific inhibitor ONX 0914 reverses susceptibility to acute viral myocarditis. EMBO Mol Med 2018, 10:200-218

23. Farini A, Sitzia C, Cassani B, Cassinelli L, Rigoni R, Colleoni F, Fusco N, Gatti S, Bella P, Villa C, Napolitano F, Maiavacca R, Bosari S, Villa A, Torrente Y: Therapeutic potential of immunoproteasome inhibition in Duchenne muscular dystrophy. Mol Ther 2016, 24:1898-1912

24. Lian Q, Zhang Y, Zhang J, Zhang HK, Wu X, Zhang Y, Lam FF, Kang S, Xia JC, Lai WH, Au KW, Chow YY, Siu CW, Lee CN, Tse HF: Functional mesenchymal stem cells derived from human induced pluripotent stem cells attenuate limb ischemia in mice. Circulation 2010, 121:1113-1123

25. Parolini D, Meregalli M, Belicchi M, Razini P, Lopa R, Del Carlo B, Farini A, Maciotta S, Bresolin N, Porretti L, Pellegrino M, Torrente Y: CD20-related signaling pathway is differently activated in normal and dystrophic circulating CD133(+) stem cells. Cell Mol Life Sci 2009, 66:697-710

26. Sitzia C, Farini A, Colleoni F, Fortunato F, Razini P, Erratico S, Tavelli A, Fabrizi F, Belicchi M, Meregalli M, Comi G, Torrente Y: Improvement of endurance of DMD animal model using natural polyphenols. Biomed Res Int 2015, 2015:680615

27. Bresolin N, Zeviani M, Bonilla E, Miller RH, Leech RW, Shanske S, Nakagawa M, DiMauro S: Fatal infantile cytochrome c oxidase deficiency: decrease of immunologically detectable enzyme in muscle. Neurology 1985, 35:802-812

28. Colatsky T, Fermini B, Gintant G, Pierson JB, Sager P, Sekino Y, Strauss DG, Stockbridge N: The comprehensive in vitro proarrhythmia assay (CiPA) initiative - update on progress. J Pharmacol Toxicol Methods 2016, 81:15-20

29. Behera R, Kumar V, Lohite K, Karnik S, Kundu GC: Activation of JAK2/STAT3 signaling by osteopontin promotes tumor growth in human breast cancer cells. Carcinogenesis 2010, 31:192-200

30. Ferrington DA, Gregerson DS: Immunoproteasomes: structure, function, and antigen presentation. Prog Mol Biol Transl Sci 2012 , 109:75-112

31. Pickering AM, Koop AL, Teoh CY, Ermak G, Grune T, Davies KJ: The immunoproteasome, the 20S proteasome and the PA28alphabeta proteasome regulator are oxidative-stress-adaptive proteolytic complexes. Biochem J 2010, 432:585-594
32. Kuswanto W, Burzyn D, Panduro M, Wang KK, Jang YC, Wagers AJ, Benoist C, Mathis D: Poor repair of skeletal muscle in aging mice reflects a defect in local, interleukin-33-dependent accumulation of regulatory T cells. Immunity 2016, 44:355-367

33. Cole MA, Rafael JA, Taylor DJ, Lodi R, Davies KE, Styles P: A quantitative study of bioenergetics in skeletal muscle lacking utrophin and dystrophin. Neuromuscul Disord 2002, 12:247-257

34. Vila MC, Rayavarapu S, Hogarth MW, Van der Meulen JH, Horn A, Defour A, Takeda S, Brown KJ, Hathout Y, Nagaraju K, Jaiswal JK Mitochondria mediate cell membrane repair and contribute to Duchenne muscular dystrophy. Cell Death Differ 2017, 24:330-342

35. Kyrychenko V, Polakova E, Janicek R, Shirokova N: Mitochondrial dysfunctions during progression of dystrophic cardiomyopathy. Cell Calcium 2015, 58:186-195

36. Shirokova N, Niggli E: Cardiac phenotype of Duchenne muscular dystrophy: insights from cellular studies. J Mol Cell Cardiol 2013, 58: 217-224

37. Okita K, Matsumura Y, Sato Y, Okada A, Morizane A, Okamoto S, Hong H, Nakagawa M, Tanabe K, Tezuka K, Shibata T, Kunisada T, Takahashi M, Takahashi J, Saji H, Yamanaka S: A more efficient method to generate integration-free human iPS cells. Nat Methods 2011, 8:409-412

38. Spaltro G, Vigorelli V, Casalnuovo F, Spinelli P, Castiglioni E, Rovina D, Paganini S, Di Segni M, Nigro P, Gervasini C, Pompilio G, Gowran A: Derivation of the Duchenne muscular dystrophy patient-derived induced pluripotent stem cell line lacking DMD exons 49 and 50 (CCMi001DMD-A-3, 49, 50). Stem Cell Res 2017, 25:128-131

39. Izumi-Nakaseko H, Nakamura $\mathrm{Y}$, Wada T, Ando K, Kanda Y, Sekino Y, Sugiyama A: Characterization of human iPS cell-derived cardiomyocyte sheets as a model to detect drug-induced conduction disturbance. J Toxicol Sci 2017, 42:183-192

40. Nakamura Y, Matsuo J, Miyamoto N, Ojima A, Ando K, Kanda Y, Sawada K, Sugiyama A, Sekino Y: Assessment of testing methods for drug-induced repolarization delay and arrhythmias in an iPS cellderived cardiomyocyte sheet: multi-site validation study. J Pharmacol Sci 2014, 124:494-501

41. Nanni S, Re A, Ripoli C, Gowran A, Nigro P, D'Amario D, Amodeo A, Crea F, Grassi C, Pontecorvi A, Farsetti A, Colussi C: The nuclear pore protein Nup153 associates with chromatin and regulates cardiac gene expression in dystrophic mdx hearts. Cardiovasc Res 2016, 112:555-567

42. Markham LW, Kinnett K, Wong BL, Woodrow Benson D, Cripe LH: Corticosteroid treatment retards development of ventricular dysfunction in Duchenne muscular dystrophy. Neuromuscul Disord 2008, 18:365-370

43. Marques MJ, Oggiam DS, Barbin IC, Ferretti R, Santo Neto H: Longterm therapy with deflazacort decreases myocardial fibrosis in $\mathrm{mdx}$ mice. Muscle Nerve 2009, 40:466-468

44. Spurney CF, Knoblach S, Pistilli EE, Nagaraju K, Martin GR, Hoffman EP: Dystrophin-deficient cardiomyopathy in mouse: expression of Nox 4 and Lox are associated with fibrosis and altered functional parameters in the heart. Neuromuscul Disord 2008, 18:371-381

45. Chen S, Kammerl IE, Vosyka O, Baumann T, Yu Y, Wu Y, Irmler M, Overkleeft HS, Beckers J, Eickelberg O, Meiners S, Stoeger T: Immunoproteasome dysfunction augments alternative polarization of alveolar macrophages. Cell Death Differ 2016, 23:1026-1037

46. Yan W, Bi HL, Liu LX, Li NN, Liu Y, Du J, Wang HX, Li HH: Knockout of immunoproteasome subunit beta2i ameliorates cardiac fibrosis and inflammation in DOCA/Salt hypertensive mice. Biochem Biophys Res Commun 2017, 490:84-90

47. Vetrone SA, Montecino-Rodriguez E, Kudryashova E, Kramerova I, Hoffman EP, Liu SD, Miceli MC, Spencer MJ: Osteopontin promotes fibrosis in dystrophic mouse muscle by modulating immune cell subsets and intramuscular TGF-beta. J Clin Invest 2009, 119:1583-1594

48. Dahiya S, Givvimani S, Bhatnagar S, Qipshidze N, Tyagi SC, Kumar A: Osteopontin-stimulated expression of matrix 
metalloproteinase-9 causes cardiomyopathy in the mdx model of Duchenne muscular dystrophy. J Immunol 2011, 187:2723-2731

49. Raman SV, Hor KN, Mazur W, Halnon NJ, Kissel JT, He X, Tran T, Smart S, McCarthy B, Taylor MD, Jefferies JL, Rafael-Fortney JA, Lowe J, Roble SL, Cripe LH: Eplerenone for early cardiomyopathy in Duchenne muscular dystrophy: a randomised, double-blind, placebocontrolled trial. Lancet Neurol 2015, 14:153-161

50. Kharraz Y, Guerra J, Mann CJ, Serrano AL, Munoz-Canoves P: Macrophage plasticity and the role of inflammation in skeletal muscle repair. Mediators Inflamm 2013, 2013:491497
51. Bauer R, Straub V, Blain A, Bushby K, MacGowan GA: Contrasting effects of steroids and angiotensin-converting-enzyme inhibitors in a mouse model of dystrophin-deficient cardiomyopathy. Eur J Heart Fail 2009, 11:463-471

52. Guerron AD, Rawat R, Sali A, Spurney CF, Pistilli E, Cha HJ, Pandey GS, Gernapudi R, Francia D, Farajian V, Escolar DM, Bossi L, Becker M, Zerr P, de la Porte S, Gordish-Dressman H, Partridge T, Hoffman EP, Nagaraju K: Functional and molecular effects of arginine butyrate and prednisone on muscle and heart in the mdx mouse model of Duchenne Muscular Dystrophy. PLoS One 2010, 5:e11220 